\title{
Bayesian Analysis of 3-Component Unit Lindley Mixture Model with Application to Extreme Observations
}

\author{
Maryam Khalid $\mathbb{D}^{1,2}$ and Muhammad Aslam ${ }^{1}$ \\ ${ }^{1}$ Department of Mathematics and Statistics, Riphah International University, Islamabad, Pakistan \\ ${ }^{2}$ Department of Statistics, Quaid I Azam University, Islamabad, Pakistan \\ Correspondence should be addressed to Maryam Khalid; m.aslam@riphah.edu.pk
}

Received 6 June 2021; Accepted 13 January 2022; Published 27 February 2022

Academic Editor: Ibrahim Almanjahie

Copyright ( 2022 Maryam Khalid and Muhammad Aslam. This is an open access article distributed under the Creative Commons Attribution License, which permits unrestricted use, distribution, and reproduction in any medium, provided the original work is properly cited.

\begin{abstract}
Bayesian inference of the 3-component unit Lindley right censored mixture is presented in this paper. The posterior distributions of the parameters are derived assuming informative (gamma) as well as noninformative (uniform and Jeffreys) priors. For the gamma (informative) prior, hyperparameters are elicited using prior predictive distribution. The Bayesian estimation has been carried out considering both symmetric and asymmetric loss functions (squared error, quadratic, weighted, and precautionary loss functions). Simulation studies for various sample sizes and different threshold values (test termination times) are considered to evaluate the performances of the Bayes estimators w.r.t their posterior risks under the said loss functions. Real life flood data from Naser Lake is also analyzed as a 3-component mixture for the sake of illustrative purpose. The simulation study and data analysis reveals that the estimates under informative prior perform better than the noninformative priors. Also, it is observed that the increase in sample size and the threshold value (test termination time) are inversely proportional to the posterior risks. Among the loss functions considered, the loss functions performance from the best to the least, w.r.t the posterior risk, is as follows: precautionary loss function < weighted loss function < quadratic loss function $<$ squared error loss function. Posterior risks are directly proportional to the sample size and threshold value (test termination time).
\end{abstract}

\section{Introduction}

Despite the advent of computational and numerical techniques, complexity of beta model has always been an issue that hindered the extensive analysis of variables bounded over the $[0,1]$ region. The quest to find a better and comparatively less cumbersome probability model led the researchers towards Kumaraswamy distribution introduced by Kumaraswamy [1]. The pursuit of a better and less complicated model led to the single parameter unit Lindley distribution proposed by Mazucheli et al. [2] as an alternate to analyze the life time data dealing with proportions, indices, fractions, or the characteristics bounded over the unit interval. The analysis of statistical properties demonstrated that it yielded better fit than the most widely used beta distribution. They further established that unit Lindley distribution is the only single parameter distribution, amongst all the distributions bounded over interval $(0,1)$, which is unimodal and has closed form expressions for c.d.f, moments, and quantiles. Biswas and Chakraborty [3] have discussed the parameter estimation of the unit Lindley distribution using Bayesian approach and its application in public health domain while Nadarajah and Chan [4] have derived the closed forms expressions for the moments of unit Lindley distribution. Alton and Cordeiro [5] proposed a unit-improved second degree Lindley distribution and analyzed its structural properties. Akdur [6] discussed parameter estimation unit Lindley mixed effect model in case of clustered and longitudinal proportion data.

Extreme value models are typically used to describe the distribution of rare events and a plethora of recent articles have proposed various extreme value mixture models for threshold estimation and quantifying the corresponding uncertainty (see Frigessi et al. [7], Do Nascimento et al. [8], 
and Jagtap and Naik-Nimbalkar [9]). The convenience of employing mixture models to extreme value data essentially arises from the ease with which multiple datasets and forecasting situations can be premeditated using such models.

Mixture distributions provide probabilistic models for populations where the overall population comprises of a mixture of several subpopulations. Mixture models facilitate the use of versatile modeling in real life areas, where only simple models were considered until the end of the twentieth century. The flexibility of mixture models has proliferated their exploitation as a convenient and semiparametric tool to model unknown distributional shapes. In addition to that mixture models are popular where there are group-structures in the data or where the aim is to explore the data for such structure, as in a cluster analysis. Sultan et al. [10]; Hassan et al. [11]; Feroze and Aslam [12]; Al-Moisheer et al. [13]; and Aslam et al. [14] have all provided analysis of 2component mixtures distributions under different censoring schemes. Most of the previous analysis of finite mixture models, Bayesian or otherwise, considered 2-component mixtures. However, the life testing experiments are very much similar to real life where things are not as simple as black and white. For instance, when modeling the price of a randomly chosen book, we need to realize the availability of book types and their prices. Before the dawn of online resources, only two types of books used to be available to readers: the paperback and hardback books. The paperback are comparatively cheaper than hardbacks. Therefore, a 2component mixture distribution can sufficiently model the prices. However, in today's era, online books are also available to readers apart from paperbacks and hardbacks. Therefore, a 3-component mixture model instead of 2component mixture will be more adequate. Many researchers have attempted to analyze the 3-component mixtures. For instance, Cheema et al. [15] investigated the 3component mixture of exponentiated inverted Weibull distribution. Khalid et al. [16] analyzed the 3-component Kumaraswamy mixture model.

Motivated by the previous research on 3-component mixture distributions, we examine the 3-component unit Lindley mixture under Bayesian inference. The remaining paper is designed as follows: Section 2 provides the mathematical basis of the mixture model, whereas Section 3 discusses the posterior distribution of parameters under gamma (informative) prior and uniform and Jeffreys (noninformative) priors along with the elicitation of hyperparameters via prior predictive distribution. The derived expressions for Bayes estimates (BEs) and their respective posterior risks (PRs), under the aforementioned loss functions, are presented in Section 4. Sections 5 and 6 provide the results of simulation study and real life data analysis. Finally, the results of the analysis are concluded in Section 7.

\section{Sampling and Likelihood Function}

Let us suppose a population comprises of 3 subpopulations with the unknown component parameters $\alpha_{l}, l=1,2,3$ and mixing weights parameters $\left\{\lambda_{1}, \lambda_{2}, \lambda_{3}\right\}$, then the resulting density function for 3-component mixture of a unit Lindley distribution (referred to as 3-CULD afterwards) is

$$
\begin{aligned}
f(x)= & \lambda_{1} \frac{\alpha_{1}}{1+\alpha_{1}}(1-x)^{-3} e^{-\alpha_{1} x / 1-x} \\
& +\lambda_{2} \frac{\alpha_{2}}{1+\alpha_{2}}(1-x)^{-3} e^{-\alpha_{2} x / 1-x} \\
& +\left(1-\lambda_{1}-\lambda_{2}\right) \frac{\alpha_{3}}{1+\alpha_{3}}(1-x)^{-3} e^{-\alpha_{3} x / 1-x}, \\
0 \leq x \leq 1, \alpha_{l}>0, l= & 1,2,3,0<\lambda_{l}<1, \sum_{l=1}^{3} \lambda_{l}=1 .
\end{aligned}
$$

Also, the distribution function is

$$
F(x)=1-\sum_{l=1}^{3} \lambda_{l}\left\{1+\frac{\alpha_{l} x}{\left(1+\alpha_{l}\right)(1-x)}\right\} e^{-\alpha_{1} x / 1-x} .
$$

Suppose a random sample of size " $n$ " is selected from unit Lindley distribution and is set up for life expectancy investigation. Suppose $r$ elements out of $n$ have the lifespan $\left\{0, \gamma_{0}\right\}$. Here, $\gamma_{0}$ is the planned time to discontinue the experiment. The remaining element " $n-r$ " objects are still active at the discontinuation of the experiment. Furthermore, each subpopulation has a total of $n_{l}$ units out of which $r_{l}$ units have deteriorated. Thus, $r=\sum_{l=1}^{3} r_{l}$ and $n=\sum_{l=1}^{3} n_{l}$. Now, suppose $x_{l k}$ represents the deteriorated time of $k^{t h}$ unit associated with $l^{t h}$ subpopulation. Here, $k=1,2, \ldots, r_{l}$, $0<x_{l k}<\gamma_{0}$, and $l=1,2,3$. The corresponding likelihood function of the observed data under Type-I censoring is thus given as

$$
\begin{aligned}
& L(\Omega \mid x) \propto \prod_{i=1}^{r_{1}} \lambda_{1} \frac{\alpha_{1}}{1+\alpha_{1}}(1-x)^{-3} e^{-\left(\alpha_{1} x / 1-x\right)} \prod_{i=1}^{r_{2}} \lambda_{2} \frac{\alpha_{2}}{1+\alpha_{2}}(1-x)^{-3} e^{-\alpha_{2} x / 1-x} \\
& \prod_{i=1}^{r_{3}} \lambda_{3} \frac{\alpha_{3}}{1+\alpha_{3}}(1-x)^{-3} e^{-\left(\alpha_{1} x / 1-x\right)}\left\{\sum_{l=1}^{3} \lambda_{l}\left(1+\frac{\alpha_{l} \gamma_{0}}{\left(1+\alpha_{l}\right)\left(1-\gamma_{0}\right)}\right) e^{-\left(\alpha_{l} \gamma_{0} / 1-\gamma_{0}\right)}\right\}^{n-r} \\
& L(\Omega \mid x) \propto \sum_{j=0}^{n-r} \sum_{m=0}^{j} \sum_{m_{1}=0}^{n-r-j} \sum_{m_{2}=0}^{j-m_{1}} \sum_{m_{3}=0}^{m_{1}} \Phi \lambda_{1}^{r_{1}+n-r-j} \lambda_{2}^{r_{2}+j-m}\left(1-\lambda_{1}\right)^{r_{3}+j} \prod_{k=1}^{3} \frac{\alpha_{k}^{2 r_{k}+m_{k}}}{\left(1+\alpha_{k}\right)^{r_{k}+m_{k}}} e^{-\alpha_{k}\left\{\psi_{k}(x)\right\}},
\end{aligned}
$$


where

$$
\begin{aligned}
& \Omega=\left\{\alpha_{1}, \alpha_{2}, \alpha_{3}, \lambda_{1}, \lambda_{2}\right\}, \\
& \Phi=\left(\begin{array}{c}
n-r \\
j
\end{array}\right)\left(\begin{array}{c}
j \\
m
\end{array}\right)\left(\begin{array}{c}
n-r-j \\
m_{1}
\end{array}\right) \\
& \left(\begin{array}{c}
j-m_{1} \\
m_{2}
\end{array}\right)\left(\begin{array}{l}
m_{1} \\
m_{3}
\end{array}\right)\left(\frac{\gamma_{0}}{1-\gamma_{0}}\right)^{m_{1}+m_{2}+m_{3}}, \\
& \psi_{1}(x)=\sum_{1}^{r_{1}} \frac{x}{1-x}+\frac{(n-r-j) \gamma_{0}}{1-\gamma_{0}}, \\
& \psi_{2}(x)=\sum_{1}^{r_{2}} \frac{x}{1-x}+\frac{(j-m) \gamma_{0}}{1-\gamma_{0}}, \\
& \psi_{3}(x)=\sum_{1}^{r_{3}} \frac{x}{1-x}+\frac{j \gamma_{0}}{1-\gamma_{0}} \text {. }
\end{aligned}
$$

\section{Bayesian Estimation for 3-CULD}

3.1. Posterior Distribution Assuming Informative Prior. The informative priors assumed for component parameters $\alpha_{l}$ and mixing parameters $\left(\lambda_{1}, \lambda_{2}\right)$ are gamma $\left(a_{l}, b_{l}\right)$ and Dirichlet $(a, b, c)$, respectively. The joint prior distribution is thus formulated as

$$
\pi_{g}(\Omega) \propto \lambda_{1}^{a-1} \lambda_{2}^{b-1} \lambda_{3}^{c-1} \alpha_{1}^{a_{1}-1} e^{-b_{1} \alpha_{1}} \alpha_{2}^{a_{2}-1} e^{-b_{2} \alpha_{2}} \alpha_{32}^{a_{3}-1} e^{-b_{3} \alpha_{3}} .
$$

The posterior distribution is thus formulated as

$$
\begin{aligned}
p_{g}(\Omega \mid x)= & \frac{1}{\Lambda_{g}} \sum_{j=0}^{n-r} \sum_{m=0}^{j} \sum_{m_{1}=0}^{n-r-j} \sum_{m_{2}=0}^{j-m_{1}} \sum_{m_{3}=0}^{m_{1}} \Phi \prod_{k=1}^{3} \lambda_{k}^{v_{g k}-1} \\
& \frac{\alpha_{k}^{\gamma_{g k}-1}}{\left(1+\alpha_{k}\right)^{r_{k}+m_{k}}} e^{-\alpha_{k}\left\{\psi_{k}(x)+b_{k}\right\}}
\end{aligned}
$$

where

$$
\begin{aligned}
\Lambda_{g}= & \sum_{j=0}^{n-r} \sum_{m=0}^{j} \sum_{m_{1}=0}^{n-r-j} \sum_{m_{2}=0}^{j-m_{1}} \sum_{m_{3}=0}^{m_{1}} \Phi B\left(v_{g 1}, v_{g 2}+v_{g 3}\right) B \\
& \quad\left(v_{g 2}, v_{g 1}+v_{g 3}\right) \prod_{k=1}^{3} \int_{\alpha_{k}} \frac{\alpha_{k}^{\gamma_{g k}-1}}{\left(1+\alpha_{k}\right)^{r_{k}+m_{k}}} e^{-\alpha_{k} \delta_{g k}} \mathrm{~d} \alpha_{k}, \\
v_{g 1} & =r_{1}+n-r-j+a \\
v_{g 2}= & r_{2}+j-m+b \\
v_{g 3}= & r_{3}+j+c \\
\gamma_{g s}= & 2 r_{s}+m_{s}+a_{s} \\
\delta_{g s} & =\psi_{s}(x)+b_{s} .
\end{aligned}
$$

Also, the respective marginal posterior densities are

$$
\begin{aligned}
& p_{g}\left(\alpha_{s} \mid x\right)=\frac{1}{\Lambda_{g}} \sum_{j=0}^{n-r} \Phi_{g}(s) \frac{\alpha_{s}^{\gamma_{g s}-1}}{\left(1+\alpha_{s}\right)^{r_{s}+m_{s}}} e^{-\alpha_{s} \delta_{g s},} \\
& p_{g}\left(\lambda_{1} \mid x\right)=\frac{1}{\Lambda_{g}} \sum_{j=0}^{n-r} \Phi_{g 1} \lambda_{1}^{v_{g 1}-1}\left(1-\lambda_{1}\right)^{v_{g 2}+v_{g 3}-1}, \\
& p_{g}\left(\lambda_{2} \mid x\right)=\frac{1}{\Lambda_{g}} \sum_{j=0}^{n-r} \Phi_{g 2} \lambda_{2}^{v_{g 2}-1}\left(1-\lambda_{2}\right)^{v_{g 1}+v_{g 3}-1},
\end{aligned}
$$

where $s=1,2,3$,

$$
\begin{aligned}
\Phi_{g}(s) & =\sum_{m=0}^{j} \sum_{m_{1}=0}^{n-r-j} \sum_{m_{2}=0}^{j-m_{1}} \sum_{m_{3}=0}^{m_{1}} \Phi B\left(v_{g 1}, v_{g 2}+v_{g 3}\right) B\left(v_{g 2}, v_{g 1}+v_{g 3}\right) \prod_{\substack{k=1 \\
k \neq s}}^{3} \int_{\alpha_{k}} \frac{\alpha_{k}^{\gamma_{g k}-1}}{\left(1+\alpha_{k}\right)^{r_{k}+m_{k}}} e^{-\alpha_{k} \delta_{g k}} \mathrm{~d} \alpha_{k}, \\
\Phi_{g 1} & =\sum_{m=0}^{j} \sum_{m_{1}=0}^{n-r-j} \sum_{m_{2}=0}^{j-m_{1}} \sum_{m_{3}=0}^{m_{1}} \Phi B\left(v_{g 2}, v_{g 1}+v_{g 3}\right) \prod_{\substack{k=1 \\
k \neq s}}^{3} \int_{\alpha_{k}} \frac{\alpha_{k}^{\gamma_{g k}-1}}{\left(1+\alpha_{k}\right)^{r_{k}+m_{k}}} e^{-\alpha_{k} \delta_{g k}} \mathrm{~d} \alpha_{k}, \\
\Phi_{g 2} & =\sum_{m=0}^{j} \sum_{m_{1}=0}^{n-r-j} \sum_{m_{2}=0}^{j-m_{1}} \sum_{m_{3}=0}^{m_{1}} \Phi B\left(v_{g 1}, v_{g 2}+v_{g 3}\right) \prod_{\substack{k=1 \\
k \neq s}}^{3} \int_{\alpha_{k}} \frac{\alpha_{k}^{\gamma_{g k}-1}}{\left(1+\alpha_{k}\right)^{r_{k}+m_{k}}} e^{-\alpha_{k} \delta_{g k}} \mathrm{~d} \alpha_{k} .
\end{aligned}
$$


It is evident from (7)-(9) that the marginal posterior distributions cannot be reduced to closed forms. Therefore, numerical quadrature techniques will be used to evaluate them.

3.1.1. Elicitation of Hyperparameters. Elicitation is one of the most important tools in Bayesian paradigm that helps in quantifying the prior knowledge and belief of a person. Despite the fact that elicitation has been a challenging task, many researchers have attempted to address elicitation problem and numerous methods for elicitation have been designed. Kadane [17]; Gartwaite and Dickey [18]; Aslam [19]; and Hahn [20] have discussed the elicitation problem in detail. Aslam [19] proposed different methods of elicitation of hyperparameters and in particular suggested the use of prior predictive distribution for the sake of elicitation. The method has become quite popular among the researchers and is frequently used for the elicitation. We have used the prior predictive distribution to estimate the hyperparameters via method of elicitation as suggested by Aslam [19]. The method is based on the confidence levels for particular intervals, where the confidence levels are the probabilities for given intervals. The following function is then used to elicit the hyperparameters $\left(a_{1}, a_{2}, \ldots, a_{m}\right)$.

$$
\xi\left(a_{1}, a_{2}, \ldots, a_{k}\right)=\min _{a_{1}, a_{2}, \ldots, a_{k}} \sum_{m=1}^{h}\left|(C C L)_{m}-(E C L)_{m}\right|,
$$

where $h$ is the number of intervals considered for elicitation, CCL represents the confidence level described by the hyperparameters, and ECL denotes the elicited confidence level. The set with minimum value of $\xi\left(a_{1}, a_{2}, \ldots, a_{k}\right)$ is considered as the elicited values of hyperparameters. The prior predictive distribution for 3-CULD is

$$
f(y)=\frac{\Gamma(a+c) \Gamma(b+c)(1-x)^{-3}}{(a+b+c)^{2} \Gamma(c)}\left\{\begin{array}{c}
\frac{a(a+c) b_{1}^{a_{1}}}{\Gamma\left(a_{1}\right)} \int_{0}^{1} \frac{\alpha_{1}^{a_{1}+1}}{1+\alpha_{1}} e^{-\left((x / 1-x)+b_{1}\right) \alpha_{1}} d \alpha_{1}+\frac{b(b+c) b_{2}^{a_{2}}}{\Gamma\left(a_{2}\right)} \int_{0}^{2} \frac{\alpha_{2}^{a_{2}+1}}{1+\alpha_{2}} e^{-\alpha_{2}\left((x / 1-x)+b_{2}\right)} \mathrm{d} \alpha_{2} \\
+\frac{(a+c)(b+c) b_{3}^{a_{3}}}{\Gamma\left(a_{3}\right)} \int_{0}^{3} \frac{\alpha_{3}^{a_{3}+1}}{1+\alpha_{3}} e^{-\alpha_{3}\left(x / 1-x+b_{3}\right)} \mathrm{d} \alpha_{3}
\end{array}\right\}
$$

Although the prior predictive distribution in (13) is not in closed form, however, we can use its numerical solution to elicit the hyperparameters. Using the prior predictive in (13), we consider the following nine intervals and their respective probabilities $\left(x_{0}, x_{m}, p_{i}\right)$ for elicitation purpose:

$$
\begin{aligned}
& (0.6,0.7 ; 0.15),(0.45,0.6 ; 0.15),(0.7,0.8 ; 0.20), \\
& (0.75,0.85 ; 0.25),(0.8,0.85 ; 0.20),(0.85,0.9 ; 0.25) .
\end{aligned}
$$

The aforementioned confidence levels for the prior predictive distribution may be elicited by solving (15) simultaneously for nine intervals via "PROC SYSLIN" in the SAS package for eliciting hyperparameters $\left(a, b, c, a_{1}, b_{1}, a_{2}, b_{2}, a_{3}, b_{3}\right)$.

$$
p_{i}=\int_{x_{0}}^{x_{m}} f(y) \mathrm{d} y .
$$

The elicited values of the hyperparameters are found to be $a_{3}=3.9657, b_{3}=0.7373$.

\subsection{Posterior Distribution Assuming Uniform Prior.} Assuming uniform prior (UP), the joint prior is

$$
\pi_{u}(\Omega) \propto 1
$$

The joint posterior distribution assuming UP is

$$
\begin{aligned}
p_{u}(\Omega \mid x)= & \frac{1}{\Lambda_{u}} \sum_{j=0}^{n-r} \sum_{m=0}^{j} \sum_{m_{1}=0}^{n-r-j} \sum_{m_{2}=0}^{j-m_{1}} \sum_{m_{3}=0}^{m_{1}} \Phi \lambda_{1}^{v_{1}} \lambda_{2}^{v_{2}} \lambda_{3}^{v_{3}} \prod_{k=1}^{3} \\
& \frac{\alpha_{s}^{2 r_{s}+m_{s}}}{\left(1+\alpha_{s}\right)^{r_{s}+m_{k s}}} e^{-\alpha_{k}\left\{\psi_{k}(x)\right\}},
\end{aligned}
$$

where

$$
\begin{aligned}
\Lambda_{u}= & \sum_{j=0}^{n-r} \sum_{m=0}^{j} \sum_{m_{1}=0}^{n-r-j} \sum_{m_{2}=0}^{j-m_{1}} \sum_{m_{3}=0}^{m_{1}} \Phi B\left(v_{1}+1, v_{2}+v_{3}+2\right) B \\
& \left(v_{2}+1, v_{1}+v_{3}+2\right) \prod_{k=1}^{3} \int_{\alpha_{k}} \frac{\alpha_{s}^{2 r_{s}+m_{s}}}{\left(1+\alpha_{s}\right)^{r_{s}+m_{k s}}} e^{-\alpha_{k}\left\{\psi_{k}(x)\right\}} \mathrm{d} \alpha_{k}, \\
v_{1}= & r_{1}+n-r-j \\
v_{2}= & r_{2}+j-m, \\
v_{3}= & r_{3}+j, \\
\gamma_{s}= & 2 r_{s}+m_{s} .
\end{aligned}
$$

Also, the respective marginal posterior densities are 


$$
\begin{aligned}
& p_{u}\left(\alpha_{s} \mid x\right)=\frac{1}{\Lambda_{u}} \sum_{j=0}^{n-r} \Phi_{u}(s) \frac{\alpha_{s}^{2 r_{s}+m_{s}}}{\left(1+\alpha_{s}\right)^{r_{s}+m_{k s}}} e^{-\alpha_{s}\left\{\psi_{s}(x)\right\}}, \\
& p_{u}\left(\lambda_{1} \mid x\right)=\frac{1}{\Lambda_{u}} \sum_{j=0}^{n-r} \Phi_{u 1} \lambda_{1}^{v_{1}}\left(1-\lambda_{1}\right)^{v_{2}+v_{3}+1}, \\
& p_{u}\left(\lambda_{2} \mid x\right)=\frac{1}{\Lambda_{u}} \sum_{j=0}^{n-r} \Phi_{u 2} \lambda_{2}^{v_{2}}\left(1-\lambda_{1}\right)^{v_{1}+v_{3}+1},
\end{aligned}
$$

where $s=1,2,3$,

$$
\begin{aligned}
\Phi_{u}(s) & =\sum_{m=0}^{j} \sum_{m_{1}=0}^{n-r-j} \sum_{m_{2}=0}^{j-m_{1}} \sum_{m_{3}=0}^{m_{1}} \Phi B\left(v_{1}+1, v_{2}+v_{3}+2\right) B\left(v_{2}+1, v_{1}+v_{3}+2\right) \prod_{\substack{k=1 \\
k \neq s}}^{3} \int_{\alpha_{k}} \frac{\alpha_{k}^{2 r_{k}+m_{k}}}{\left(1+\alpha_{k}\right)^{r_{k}+m_{k}}} e^{-\alpha_{k}\left\{\psi_{k}(x)\right\}} \mathrm{d} \alpha_{k}, \\
\Phi_{u 1} & =\sum_{m=0}^{j} \sum_{m_{1}=0}^{n-r-j} \sum_{m_{2}=0}^{j-m_{1}} \sum_{m_{3}=0}^{m_{1}} \Phi B\left(v_{2}+1, v_{1}+v_{3}+2\right) \prod_{k=1}^{3} \int_{\alpha_{k}} \frac{\alpha_{k}^{2 r_{k}+m_{k}}}{\left(1+\alpha_{k}\right)^{r_{k}+m_{k}}} e^{-\alpha_{k}\left\{\psi_{k}(x)\right\}} \mathrm{d} \alpha_{k} \\
\Phi_{u 2} & =\sum_{m=0}^{j} \sum_{m_{1}=0}^{n-r-j} \sum_{m_{2}=0}^{j-m_{1}} \sum_{m_{3}=0}^{m_{1}} \Phi B\left(v_{1}+1, v_{2}+v_{3}+2\right) \prod_{k=1}^{3} \int_{\alpha_{k}} \frac{\alpha_{k}^{2 r_{k}+m_{k}}}{\left(1+\alpha_{k}\right)^{r_{k}+m_{k}}} e^{-\alpha_{k}\left\{\psi_{k}(x)\right\}} \mathrm{d} \alpha_{k} .
\end{aligned}
$$

3.3. Posterior Distribution Assuming Jeffreys Prior. The study assumes Jeffreys prior (JP) for the component parameters and UP for the mixture proportions. Mazucheli et al. [21] derived the JP for unit Lindley distribution as

$$
\pi_{J}(\Omega) \propto \prod_{k=1}^{3} \frac{\left(\alpha_{k}^{2}+4 \alpha_{k}+2\right)^{1 / 2}}{\alpha_{k}\left(1+\alpha_{k}\right)}
$$

The joint posterior distribution becomes

$$
\begin{aligned}
p_{J}(\Omega \mid x)= & \frac{1}{\Lambda_{J}} \sum_{j=0}^{n-r} \sum_{m=0}^{j} \sum_{m_{1}=0}^{n-r-j} \sum_{m_{2}=0}^{j-m_{1}} \sum_{m_{3}=0}^{m_{1}} \Phi \lambda_{1}^{v_{1}} \lambda_{2}^{v_{2}} \lambda_{3}^{v_{3}} \\
& \prod_{k=1}^{3} \frac{\alpha_{k}^{2 r_{k}+m_{k}-1}}{\left(1+\alpha_{k}\right)^{r_{k}+m_{k}+1}}\left(\alpha_{k}^{2}+4 \alpha_{k}+2\right)^{1 / 2} e^{-\alpha_{k}\left\{\psi_{k}(x)\right\}},
\end{aligned}
$$

where

$$
\begin{aligned}
\Lambda_{J}= & \sum_{j=0}^{n-r} \sum_{m=0}^{j} \sum_{m_{1}=0}^{n-r-j} \sum_{m_{2}=0}^{j-m_{1}} \sum_{m_{3}=0}^{m_{1}} \Phi B\left(v_{1}+1, v_{2}+v_{3}+2\right) B \\
& \left(v_{2}+1, v_{1}+v_{3}+2\right) \\
& \prod_{k=1}^{3} \int_{\alpha_{k}} \frac{\alpha_{k}^{2 r_{k}+m_{k}-1}}{\left(1+\alpha_{k}\right)^{r_{k}+m_{k}+1}}\left(\alpha_{k}^{2}+4 \alpha_{k}+2\right)^{1 / 2} e^{-\alpha_{k}\left\{\psi_{k}(x)\right\}} \mathrm{d} \alpha_{k} .
\end{aligned}
$$

Also, the respective marginal posterior densities are as follows:

$$
\begin{aligned}
& p_{J}\left(\alpha_{s} \mid x\right)=\frac{1}{\Lambda_{J}} \sum_{j=0}^{n-r} \Phi_{J}(s) \frac{\alpha_{s}^{2 r_{s}+m_{s}-1}\left(\alpha_{k}^{2}+4 \alpha_{s}+2\right)^{1 / 2}}{\left(1+\alpha_{k}\right)^{r_{s}+m_{s}+1}} e^{-\alpha_{s}\left\{\psi_{s}(x)\right\}}, \\
& p_{J}\left(\lambda_{1} \mid x\right)=\frac{1}{\Lambda_{J}} \sum_{j=0}^{n-r} \Phi_{J 1} \lambda_{1}^{v_{1}}\left(1-\lambda_{1}\right)^{v_{2}+v_{3}+1}, c \\
& p_{J}\left(\lambda_{2} \mid x\right)=\frac{1}{\Lambda_{J}} \sum_{j=0}^{n-r} \Phi_{J 2} \lambda_{2}^{v_{2}}\left(1-\lambda_{2}\right)^{v_{1}+v_{3}+1},
\end{aligned}
$$


where $s=1,2,3$,

$$
\begin{aligned}
\Phi_{J}(s)= & \sum_{m=0}^{j} \sum_{m_{1}=0}^{n-r-j} \sum_{m_{2}=0}^{j-m_{1}} \sum_{m_{3}=0}^{m_{1}} \Phi B\left(v_{1}+1, v_{2}+v_{3}+2\right) B\left(v_{2}+1, v_{1}+v_{3}+2\right), \\
& \prod_{k=1}^{3} \int_{\alpha_{k}} \frac{\alpha_{k}^{2 r_{k}+m_{k}-1}}{\left(1+\alpha_{k}\right)^{r_{k}+m_{k}+1}}\left(\alpha_{k}^{2}+4 \alpha_{k}+2\right)^{1 / 2} e^{-\alpha_{k}\left\{\psi_{k}(x)\right\}} \mathrm{d} \alpha_{k}, \\
\Phi_{J 1}= & \sum_{m=0}^{j} \sum_{m_{1}=0}^{n-r-j} \sum_{m_{2}=0}^{j-m_{1}} \sum_{m_{3}=0}^{m_{1}} \Phi B\left(v_{2}+1, v_{1}+v_{3}+2\right) \prod_{k=1}^{3} \int_{\alpha_{k}} \frac{\alpha_{k}^{2 r_{k}+m_{k}-1}}{\left(1+\alpha_{k}\right)^{r_{k}+m_{k}+1}}\left(\alpha_{k}^{2}+4 \alpha_{k}+2\right)^{1 / 2} e^{-\alpha_{k}\left\{\psi_{k}(x)\right\}} \mathrm{d} \alpha_{k}, \\
\Phi_{J 2}= & \sum_{m=0}^{j} \sum_{m_{1}=0}^{n-r-j} \sum_{m_{2}=0}^{j-m_{1}} \sum_{m_{3}=0}^{m_{1}} \Phi B\left(v_{1}+1, v_{2}+v_{3}+2\right) \prod_{k=1}^{3} \int_{\alpha_{k}} \frac{\alpha_{k}^{2 r_{k}+m_{k}-1}}{\left(1+\alpha_{k}\right)^{r_{k}+m_{k}+1}}\left(\alpha_{k}^{2}+4 \alpha_{k}+2\right)^{1 / 2} e^{-\alpha_{k}\left\{\psi_{k}(x)\right\}} \mathrm{d} \alpha_{k} .
\end{aligned}
$$

\section{Bayes Estimates and Posterior Risks}

The loss functions not only provide the Bayes estimates of the unknown parameters but also give an insight about the posterior risks associated with these estimates. These posterior risks can be further used to compare the Bayes estimates. The most commonly used loss functions for Bayesian estimation are squared error loss (SELF) and quadratic loss function (QLF). Both the loss functions are symmetrical, i.e., they assign equal weight to the losses occurred due to overestimation and underestimation which becomes an impractical restriction particularly when analyzing extreme value data, failure rate, or reliability. The parametric space for 3-CULD contains the mixing proportions defined on restricted space; the symmetric loss functions may provide suboptimal solutions and can be an inappropriate choice. Therefore, this study includes the two most commonly used asymmetric loss functions as well, namely, weighted loss function (WLF) and precautionary loss function (PLF). WLF assigns appropriate weights to the SELF in order to penalize the gross overestimation and gross underestimation. Norstrom [22] suggested use of PLF to avoid boundary values of parameters. PLF prevents underestimation particularly when smaller mixing proportions are being estimated.

The following table provides the Bayes estimates (BEs) and their posterior risks (PRs) under the four loss functions mentioned above.

The derived expressions for these BEs and their respective PRs under the gamma prior discussed in Table 1 are

$$
\begin{aligned}
\left(\widehat{\alpha}_{s} \mid x\right)_{\operatorname{SELF}(g)} & =\frac{1}{\Lambda_{g}} \sum_{j=0}^{n-r} \Phi_{g}(s) \int_{\alpha_{s}} \frac{\alpha_{s}^{\gamma_{g s}}}{\left(1+\alpha_{s}\right)^{r_{s}+m_{s}}} e^{-\alpha_{s} \delta_{g s}} \mathrm{~d} \alpha_{s}, \\
\Re\left(\widehat{\alpha}_{s} \mid x\right)_{\operatorname{SELF}(g)} & =\frac{1}{\Lambda_{g}} \sum_{j=0}^{n-r} \Phi_{g}(s) \int_{\alpha_{s}} \frac{\alpha_{s}^{\gamma_{g s}+1}}{\left(1+\alpha_{s}\right)^{r_{s}+m_{s}}} e^{-\alpha_{s} \delta_{g s}} \mathrm{~d} \alpha_{s}-\left(\widehat{\alpha}_{s} \mid x\right)_{S E L F(g)}^{2}, \\
\left(\hat{\lambda}_{1} \mid x\right)_{\operatorname{SELF}(g)} & =\frac{1}{\Lambda_{g}} \sum_{j=0}^{n-r} \Phi_{g 1} B\left(v_{g 1}+1, v_{g 2}+v_{g 3}\right), \\
\mathfrak{R}\left(\hat{\lambda}_{1} \mid x\right)_{\operatorname{SELF}(g)} & =\frac{1}{\Lambda_{g}} \sum_{j=0}^{n-r} \Phi_{g 1} B\left(v_{g 1}+2, v_{g 2}+v_{g 3}\right)-\left(\hat{\lambda}_{1} \mid x\right)_{\operatorname{SELF}(g)}^{2}, \\
\left(\hat{\lambda}_{2} \mid x\right)_{\operatorname{SELF}(g)} & =\frac{1}{\Lambda_{g}} \sum_{j=0}^{n-r} \Phi_{g 2} B\left(v_{g 2}+1, v_{g 1}+v_{g 3}\right),
\end{aligned}
$$




$$
\begin{aligned}
& \mathfrak{R}\left(\hat{\lambda}_{2} \mid x\right)_{\operatorname{SELF}(g)}=\frac{1}{\Lambda_{g}} \sum_{j=0}^{n-r} \Phi_{g 2} B\left(v_{g 2}+2, v_{g 1}+v_{g 3}\right)-\left(\hat{\lambda}_{2} \mid x\right)_{\operatorname{SELF}(g)}^{2} . \\
& \left(\widehat{\alpha}_{s} \mid x\right)_{Q L F(g)}=\frac{\sum_{j=0}^{n-r} \Phi_{g}(s) \int_{\alpha_{s}}\left(\alpha_{s}^{\gamma_{g s}-2} /\left(1+\alpha_{s}\right)^{r_{s}+m_{s}}\right) e^{-\alpha_{s} \delta_{g s}} \mathrm{~d} \alpha_{s}}{\sum_{j=0}^{n-r} \Phi_{g}(s) \int_{\alpha_{s}}\left(\alpha_{s}^{\gamma_{g s}-3} /\left(1+\alpha_{s}\right)^{r_{s}+m_{s}}\right) e^{-\alpha_{s} \delta_{g s}} \mathrm{~d} \alpha_{s}} \\
& \left(\widehat{\lambda}_{1} \mid x\right)_{\mathrm{Q} L F(g)}=\frac{\sum_{j=0}^{n-r} \Phi_{g 1} B\left(v_{g 1}-1, v_{g 2}+v_{g 3}\right)}{\sum_{j=0}^{n-r} \Phi_{g 1} B\left(v_{g 1}-2, v_{g 2}+v_{g 3}\right)}, \\
& \Re\left(\widehat{\alpha}_{s} \mid x\right)_{Q L F(g)}=1-\frac{\left\{\left(1 / \Lambda_{g}\right) \sum_{j=0}^{n-r} \Phi_{g}(s) \int_{\alpha_{s}}\left(\alpha_{s}^{\gamma_{g s}-2} /\left(1+\alpha_{s}\right)^{r_{s}+m_{s}}\right) e^{-\alpha_{s} \delta_{g s}} \mathrm{~d} \alpha_{s}\right\}^{2}}{\left(1 / \Lambda_{g}\right) \sum_{j=0}^{n-r} \Phi_{g}(s) \int_{\alpha_{s}}\left(\alpha_{s}^{\gamma_{g s}-3} /\left(1+\alpha_{s}\right)^{r_{s}+m_{s}}\right) e^{-\alpha_{s} \delta_{g s}} \mathrm{~d} \alpha_{s}}, \\
& \mathfrak{R}\left(\hat{\lambda}_{1} \mid x\right)_{\mathrm{QLF}(g)}=1-\frac{\left\{\left(1 / \Lambda_{g}\right) \sum_{j=0}^{n-r} \Phi_{g 1} B\left(v_{g 1}-1, v_{g 2}+v_{g 3}\right)\right\}^{2}}{\left(1 / \Lambda_{g}\right) \sum_{j=0}^{n-r} \Phi_{g 1} B\left(v_{g 1}-2, v_{g 2}+v_{g 3}\right)} \\
& \left(\hat{\lambda}_{2} \mid x\right)_{Q L F(g)}=\frac{\sum_{j=0}^{n-r} \Phi_{g 2} B\left(v_{g 2}-1, v_{g 1}+v_{g 3}\right)}{\sum_{j=0}^{n-r} \Phi_{g 2} B\left(v_{g 2}-2, v_{g 1}+v_{g 3}\right)} \\
& \mathfrak{R}\left(\hat{\lambda}_{2} \mid x\right)_{\mathrm{QLF}(g)}=1-\frac{\left\{\left(1 / \Lambda_{g}\right) \sum_{j=0}^{n-r} \Phi_{g 2} B\left(v_{g 2}-1, v_{g 1}+v_{g 3}\right)\right\}^{2}}{\left(1 / \Lambda_{g}\right) \sum_{j=0}^{n-r} \Phi_{g 2} B\left(v_{g 2}-2, v_{g 1}+v_{g 3}\right)}, \\
& \left(\widehat{\alpha}_{s} \mid x\right)_{W L F(g)}=\left\{\frac{1}{\Lambda_{g}} \sum_{j=0}^{n-r} \Phi_{g}(s) \int_{\alpha_{s}} \frac{\alpha_{s}^{\gamma_{g s}-2}}{\left(1+\alpha_{s}\right)^{r_{s}+m_{s}}} e^{-\alpha_{s} \delta_{g s}} \mathrm{~d} \alpha_{s}\right\}^{-1} \\
& \mathfrak{R}\left(\widehat{\alpha}_{s} \mid x\right)_{W L F(g)}=\frac{1}{\Lambda_{g}} \sum_{j=0}^{n-r} \Phi_{g}(s) \int_{\alpha_{s}} \frac{\alpha_{s}^{\gamma_{g s}}}{\left(1+\alpha_{s}\right)^{r_{s}+m_{s}}} e^{-\alpha_{s} \delta_{g s}} \mathrm{~d} \alpha_{s}-\left(\widehat{\alpha}_{s} \mid x\right)_{W L F(g)}, \\
& \left(\widehat{\lambda}_{1} \mid x\right)_{W L F(g)}=\left\{\frac{1}{\Lambda_{g}} \sum_{j=0}^{n-r} \Phi_{g 1} B\left(v_{g 1}-1, v_{g 2}+v_{g 3}\right)\right\}^{-1}, \\
& \mathfrak{R}\left(\hat{\lambda}_{1} \mid x\right)_{W L F(g)}=\frac{1}{\Lambda_{g}} \sum_{j=0}^{n-r} \Phi_{g 1} B\left(v_{g 1}+1, v_{g 2}+v_{g 3}\right)-\left(\hat{\lambda}_{1} \mid x\right)_{W L F(g)}, \\
& \left(\widehat{\lambda}_{2} \mid x\right)_{W L F(g)}=\left\{\frac{1}{\Lambda_{g}} \sum_{j=0}^{n-r} \Phi_{g 2} B\left(v_{g 2}-1, v_{g 1}+v_{g 3}\right)\right\}^{-1}, \\
& \mathfrak{R}\left(\hat{\lambda}_{2} \mid x\right)_{W L F(g)}=\frac{1}{\Lambda_{g}} \sum_{j=0}^{n-r} \Phi_{g 2} B\left(v_{g 2}+1, v_{g 1}+v_{g 3}\right)-\left(\hat{\lambda}_{2} \mid x\right)_{W L F(g)}, \\
& \left(\widehat{\alpha}_{s} \mid x\right)_{P L F(g)}=\left\{\frac{1}{\Lambda_{g}} \sum_{j=0}^{n-r} \Phi_{g}(s) \int_{\alpha_{s}} \frac{\alpha_{s}^{\gamma_{g s}+1}}{\left(1+\alpha_{s}\right)^{r_{s}+m_{s}}} e^{-\alpha_{s} \delta_{g s}} \mathrm{~d} \alpha_{s}\right\}^{1 / 2},
\end{aligned}
$$




$$
\begin{aligned}
\mathfrak{R}\left(\hat{\alpha}_{s} \mid x\right)_{P L F(g)} & =2\left\{\left(\widehat{\alpha}_{s} \mid x\right)_{P L F(g)}-\frac{1}{\Lambda_{g}} \sum_{j=0}^{n-r} \Phi_{g}(s) \int_{\alpha_{s}} \frac{\alpha_{s}^{\gamma_{g s}}}{\left(1+\alpha_{s}\right)^{r_{s}+m_{s}}} e^{-\alpha_{s} \delta_{g s}} \mathrm{~d} \alpha_{s}\right\}, \\
\left(\hat{\lambda}_{1} \mid x\right)_{P L F(g)} & =\left\{\frac{1}{\Lambda_{g}} \sum_{j=0}^{n-r} \Phi_{g 1} B\left(v_{g 1}+2, v_{g 2}+v_{g 3}\right)\right\}^{1 / 2}, \\
\mathfrak{R}\left(\hat{\lambda}_{1} \mid x\right)_{P L F(g)} & =2\left\{\left(\widehat{\lambda}_{1} \mid x\right)_{P L F(g)}-\frac{1}{\Lambda_{g}} \sum_{j=0}^{n-r} \Phi_{g 1} B\left(v_{g 1}+1, v_{g 2}+v_{g 3}\right)\right\}, \\
\left(\hat{\lambda}_{2} \mid x\right)_{P L F(g)} & =\left\{\frac{1}{\Lambda_{g}} \sum_{j=0}^{n-r} \Phi_{g 2} B\left(v_{g 2}+2, v_{g 1}+v_{g 3}\right)\right\}, \\
\mathfrak{R}\left(\hat{\lambda}_{2} \mid x\right)_{P L F(g)} & =2\left\{\left(\hat{\lambda}_{2} \mid x\right)_{P L F(g)}-\frac{1}{\Lambda_{g}} \sum_{j=0}^{n-r} \Phi_{g 2} B\left(v_{g 2}+1, v_{g 1}+v_{g 3}\right)\right\} .
\end{aligned}
$$

The derived expressions for these BEs and their respective PRs under the uniform prior discussed in Table 1 are

$$
\begin{aligned}
& \left(\widehat{\alpha}_{s} \mid x\right)_{S E L F(u)}=\frac{1}{\Lambda_{u}} \sum_{j=0}^{n-r} \Phi_{u}(s) \int_{\alpha_{s}} \frac{\alpha_{s}^{\gamma_{s}+1}}{\left(1+\alpha_{k}\right)^{r_{s}+m_{s}}} e^{-\alpha_{s}\left\{\psi_{s}(x)\right\}} \mathrm{d} \alpha_{s}, \\
& \Re\left(\widehat{\alpha}_{s} \mid x\right)_{S E L F(u)}=\frac{1}{\Lambda_{u}} \sum_{j=0}^{n-r} \Phi_{u}(s) \int_{\alpha_{s}} \frac{\alpha_{s}^{\gamma_{s}+2}}{\left(1+\alpha_{k}\right)^{r_{s}+m_{s}}} e^{-\alpha_{s}\left\{\psi_{s}(x)\right\}} \mathrm{d} \alpha_{s}-\left(\widehat{\alpha}_{s} \mid x\right)_{S E L F(u)}^{2}, \\
& \left(\hat{\lambda}_{1} \mid x\right)_{\operatorname{SELF}(u)}=\frac{1}{\Lambda_{u}} \sum_{j=0}^{n-r} \Phi_{u 1} B\left(v_{1}+2, v_{2}+v_{3}+2\right), \\
& \mathfrak{R}\left(\hat{\lambda}_{1} \mid x\right)_{S E L F(u)}=\frac{1}{\Lambda_{u}} \sum_{j=0}^{n-r} \Phi_{u 1} B\left(v_{1}+3, v_{2}+v_{3}+2\right)-\left(\hat{\lambda}_{1} \mid x\right)_{\operatorname{SELF}(u)}^{2}, \\
& \left(\hat{\lambda}_{2} \mid x\right)_{\operatorname{SELF}(u)}=\frac{1}{\Lambda_{u}} \sum_{j=0}^{n-r} \Phi_{u 2} B\left(v_{2}+2, v_{1}+v_{3}+2\right), \\
& \mathfrak{R}\left(\widehat{\lambda}_{2} \mid x\right)_{\operatorname{SELF}(u)}=\frac{1}{\Lambda_{u}} \sum_{j=0}^{n-r} \Phi_{u 2} B\left(v_{2}+3, v_{1}+v_{3}+2\right)-\left(\widehat{\lambda}_{2} \mid x\right)_{\operatorname{SELF}(u)}^{2}, \\
& \left(\widehat{\alpha}_{s} \mid x\right)_{\mathrm{QLF}(u)}=\frac{\sum_{j=0}^{n-r} \Phi_{u}(s) \int_{\alpha_{s}}\left(\alpha_{s}^{\gamma_{s}-1} /\left(1+\alpha_{k}\right)^{r_{s}+m_{s}}\right) e^{-\alpha_{s}\left\{\psi_{s}(x)\right\}} \mathrm{d} \alpha_{s}}{\sum_{j=0}^{n-r} \Phi_{u}(s) \int_{\alpha_{s}}\left(\alpha_{s}^{\gamma_{s}-2} /\left(1+\alpha_{k}\right)^{r_{s}+m_{s}}\right) e^{-\alpha_{s}\left\{\psi_{s}(x)\right\}} \mathrm{d} \alpha_{s}}, \\
& \mathfrak{R}\left(\widehat{\alpha}_{s} \mid x\right)_{\mathrm{QLF}(u)}=1-\frac{\left\{1 / \Lambda_{u} \sum_{j=0}^{n-r} \Phi_{u}(s) \int_{\alpha_{s}}\left(\alpha_{s}^{\gamma_{s}-1} /\left(1+\alpha_{k}\right)^{r_{s}+m_{s}}\right) e^{-\alpha_{s}\left\{\psi_{s}(x)\right\}} \mathrm{d} \alpha_{s}\right\}^{2}}{1 / \Lambda_{u} \sum_{j=0}^{n-r} \Phi_{u}(s) \int_{\alpha_{s}}\left(\alpha_{s}^{\gamma_{s}-2} /\left(1+\alpha_{k}\right)^{r_{s}+m_{s}}\right) e^{-\alpha_{s}\left\{\psi_{s}(x)\right\}} \mathrm{d} \alpha_{s}},
\end{aligned}
$$


TABLE 1: Loss function and their corresponding BEs and PRs.

\begin{tabular}{lcr}
\hline Loss function & Bayes estimate $(\mathrm{BE})$ & Posterior risk (PR) \\
\hline SELF: $(\theta-d)^{2}$ & $E(\theta \mid x)$ & Var $(\theta \mid x)$ \\
QLF: $(1-d / \theta)^{2}$ & $E\left(\theta^{-1} \mid x\right) / E\left(\theta^{-2} \mid x\right)$ & $1-\left(E\left(\theta^{-1} \mid x\right)^{2} / E\left(\theta^{-2} \mid x\right)\right)$ \\
WLF: $(\theta-d)^{2} / \theta$ & $\left\{E\left(\theta^{-1} \mid x\right)\right\}^{-1}$ & $E(\theta \mid x)-\left\{E\left(\theta^{-1} \mid x\right)\right\}^{-1}$ \\
PLF: $(\theta-d)^{2} / d$ & $\sqrt{E\left(\theta^{2} \mid x\right)}$ & $2\left\{\sqrt{\left.E\left(\theta^{2} \mid x\right)-E(\theta \mid x)\right\}}\right.$ \\
\hline
\end{tabular}

$$
\begin{aligned}
& \left(\widehat{\lambda}_{1} \mid x\right)_{\mathrm{QLF}(u)}=\frac{\sum_{j=0}^{n-r} \Phi_{u 1} B\left(v_{1}, v_{2}+v_{3}+2\right)}{\sum_{j=0}^{n-r} \Phi_{u 1} B\left(v_{1}-1, v_{2}+v_{3}+2\right)}, \\
& \mathfrak{R}\left(\widehat{\lambda}_{1} \mid x\right)_{\mathrm{QLF}(u)}=1-\frac{\left\{1 / \Lambda_{u} \sum_{j=0}^{n-r} \Phi_{u 1} B\left(v_{1}, v_{2}+v_{3}+2\right)\right\}^{2}}{1 / \Lambda_{u} \sum_{j=0}^{n-r} \Phi_{u 1} B\left(v_{1}-1, v_{2}+v_{3}+2\right)}, \\
& \left(\widehat{\lambda}_{2} \mid x\right)_{Q L F(u)}=\frac{\sum_{j=0}^{n-r} \Phi_{u 2} B\left(v_{2}, v_{1}+v_{3}+2\right)}{\sum_{j=0}^{n-r} \Phi_{u 2} B\left(v_{2}-1, v_{1}+v_{3}+2\right)} \\
& \mathfrak{R}\left(\widehat{\lambda}_{2} \mid x\right)_{Q L F(u)}=1-\frac{\left\{1 / \Lambda_{u} \sum_{j=0}^{n-r} \Phi_{u 2} B\left(v_{2}, v_{1}+v_{3}+2\right)\right\}^{2}}{1 / \Lambda_{u} \sum_{j=0}^{n-r} \Phi_{u 2} B\left(v_{2}-1, v_{1}+v_{3}+2\right)}, \\
& \left(\widehat{\alpha}_{s} \mid x\right)_{W L F(u)}=\left\{\frac{1}{\Lambda_{u}} \sum_{j=0}^{n-r} \Phi_{u}(s) \int_{\alpha_{s}} \frac{\alpha_{s}^{\gamma_{s}-1}}{\left(1+\alpha_{k}\right)^{r_{s}+m_{s}}} e^{-\alpha_{s}\left\{\psi_{s}(x)\right\}} \mathrm{d} \alpha_{s}\right\}^{-1}, \\
& \mathfrak{R}\left(\widehat{\alpha}_{s} \mid x\right)_{W L F(u)}=\frac{1}{\Lambda_{u}} \sum_{j=0}^{n-r} \Phi_{u}(s) \int_{\alpha_{s}} \frac{\alpha_{s}^{\gamma_{s}+1}}{\left(1+\alpha_{k}\right)^{r_{s}+m_{s}}} e^{-\alpha_{s}\left\{\psi_{s}(x)\right\}} \mathrm{d} \alpha_{s}-\left(\widehat{\alpha}_{s} \mid x\right)_{W L F(u)}, \\
& \left(\widehat{\lambda}_{1} \mid x\right)_{W L F(u)}=\left\{\frac{1}{\Lambda_{u}} \sum_{j=0}^{n-r} \Phi_{u 1} B\left(v_{1}, v_{2}+v_{3}+2\right)\right\}^{-1}, \\
& \mathfrak{R}\left(\hat{\lambda}_{1} \mid x\right)_{W L F(u)}=\frac{1}{\Lambda_{u}} \sum_{j=0}^{n-r} \Phi_{u 1} B\left(v_{1}+2, v_{2}+v_{3}+2\right)-\left(\hat{\lambda}_{1} \mid x\right)_{W L F(u)} \\
& \left(\widehat{\lambda}_{2} \mid x\right)_{W L F(u)}=\left\{\frac{1}{\Lambda_{u}} \sum_{j=0}^{n-r} \Phi_{u 2} B\left(v_{2}, v_{1}+v_{3}+2\right)\right\}^{-1}, \\
& \mathfrak{R}\left(\widehat{\lambda}_{2} \mid x\right)_{W L F(u)}=\frac{1}{\Lambda_{u}} \sum_{j=0}^{n-r} \Phi_{u 2} B\left(v_{2}+2, v_{1}+v_{3}+2\right)-\left(\widehat{\lambda}_{2} \mid x\right)_{W L F(u)} \\
& \left(\widehat{\alpha}_{s} \mid x\right)_{P L F(u)}=\left\{\frac{1}{\Lambda_{u}} \sum_{j=0}^{n-r} \Phi_{u}(s) \int_{\alpha_{s}} \frac{\alpha_{s}^{\gamma_{s}+2}}{\left(1+\alpha_{k}\right)^{r_{s}+m_{s}}} e^{-\alpha_{s}\left\{\psi_{s}(x)\right\}} \mathrm{d} \alpha_{s}\right\}^{1 / 2}, \\
& \Re\left(\widehat{\alpha}_{s} \mid x\right)_{P L F(u)}=2\left\{\left(\widehat{\alpha}_{s} \mid x\right)_{P L F(u)}-\frac{1}{\Lambda_{u}} \sum_{j=0}^{n-r} \Phi_{u}(s) \int_{\alpha_{s}} \frac{\alpha_{s}^{\gamma_{s}+1}}{\left(1+\alpha_{k}\right)^{r_{s}+m_{s}}} e^{-\alpha_{s}\left\{\psi_{s}(x)\right\}} \mathrm{d} \alpha_{s}\right\},
\end{aligned}
$$




$$
\begin{aligned}
\left(\hat{\lambda}_{1} \mid x\right)_{P L F(u)} & =\left\{\frac{1}{\Lambda_{u}} \sum_{j=0}^{n-r} \Phi_{u 1} B\left(v_{1}+3, v_{2}+v_{3}+2\right)\right\}^{1 / 2}, \\
\mathfrak{R}\left(\hat{\lambda}_{1} \mid x\right)_{P L F(u)} & =2\left\{\left(\hat{\lambda}_{1} \mid x\right)_{P L F(u)}-\frac{1}{\Lambda_{u}} \sum_{j=0}^{n-r} \Phi_{u 1} B\left(v_{1}+2, v_{2}+v_{3}+2\right)\right\}, \\
\left(\hat{\lambda}_{2} \mid x\right)_{P L F(u)} & =\left\{\frac{1}{\Lambda_{u}} \sum_{j=0}^{n-r} \Phi_{u 2} B\left(v_{2}+3, v_{1}+v_{3}+2\right)\right\}^{1 / 2}, \\
\Re\left(\hat{\lambda}_{2} \mid x\right)_{P L F(u)} & =2\left\{\left(\hat{\lambda}_{2} \mid x\right)_{P L F(u)}-\frac{1}{\Lambda_{u}} \sum_{j=0}^{n-r} \Phi_{u 2} B\left(v_{2}+2, v_{1}+v_{3}+2\right)\right\} .
\end{aligned}
$$

The derived expressions for these BEs and their respective PRs under the Jeffreys prior discussed in Table 1 are

$$
\begin{aligned}
& \left(\widehat{\alpha}_{s} \mid x\right)_{\operatorname{SELF(J)}}=\frac{1}{\Lambda_{J}} \sum_{j=0}^{n-r} \Phi_{J}(s) \int_{\alpha_{s}} \frac{\alpha_{s}^{\gamma_{s}}\left(\alpha_{s}^{2}+4 \alpha_{s}+2\right)^{1 / 2}}{\left(1+\alpha_{k}\right)^{r_{s}+m_{s}+1}} e^{-\alpha_{s}\left\{\psi_{s}(x)\right\}} \mathrm{d} \alpha_{s}, \\
& \mathfrak{R}\left(\widehat{\alpha}_{s} \mid x\right)_{\operatorname{SELF}(J)}=\frac{1}{\Lambda_{J}} \sum_{j=0}^{n-r} \Phi_{J}(s) \int_{\alpha_{s}} \frac{\alpha_{s}^{\gamma_{s}+1}\left(\alpha_{s}^{2}+4 \alpha_{s}+2\right)^{1 / 2}}{\left(1+\alpha_{k}\right)^{r_{s}+m_{s}+1}} e^{-\alpha_{s}\left\{\psi_{s}(x)\right\}} \mathrm{d} \alpha_{s}-\left(\widehat{\alpha}_{s} \mid x\right)_{S E L F(J)}^{2}, \\
& \left(\widehat{\lambda}_{1} \mid x\right)_{\operatorname{SELF}(J)}=\frac{1}{\Lambda_{J}} \sum_{j=0}^{n-r} \Phi_{J 1} B\left(v_{1}+2, v_{2}+v_{3}+2\right), \\
& \Re\left(\hat{\lambda}_{1} \mid x\right)_{\operatorname{SELF}(J)}=\frac{1}{\Lambda_{J}} \sum_{j=0}^{n-r} \Phi_{J 1} B\left(v_{1}+3, v_{2}+v_{3}+2\right)-\left(\hat{\lambda}_{1} \mid x\right)_{S E L F(J)}^{2} \\
& \left(\widehat{\lambda}_{2} \mid x\right)_{S E L F(J)}=\frac{1}{\Lambda_{J}} \sum_{j=0}^{n-r} \Phi_{J 2} B\left(v_{2}+2, v_{1}+v_{3}+2\right), \\
& \mathfrak{R}\left(\hat{\lambda}_{2} \mid x\right)_{S E L F(J)}=\frac{1}{\Lambda_{J}} \sum_{j=0}^{n-r} \Phi_{J 2} B\left(v_{2}+3, v_{1}+v_{3}+2\right)-\left(\hat{\lambda}_{1} \mid x\right)_{S E L F(J)}^{2}, \\
& \left(\widehat{\alpha}_{s} \mid x\right)_{\mathrm{QLF}(J)}=\frac{\sum_{j=0}^{n-r} \Phi_{J}(s) \int_{\alpha_{s}}\left(\alpha_{s}^{\gamma_{s}-2}\left(\alpha_{s}^{2}+4 \alpha_{s}+2\right)^{1 / 2} /\left(1+\alpha_{k}\right)^{r_{s}+m_{s}+1}\right) e^{-\alpha_{s}\left\{\psi_{s}(x)\right\}} \mathrm{d} \alpha_{s}}{\sum_{j=0}^{n-r} \Phi_{J}(s) \int_{\alpha_{s}}\left(\alpha_{s}^{\gamma_{s}-3}\left(\alpha_{s}^{2}+4 \alpha_{s}+2\right)^{1 / 2} /\left(1+\alpha_{k}\right)^{r_{s}+m_{s}+1}\right) e^{-\alpha_{s}\left\{\psi_{s}(x)\right\}} \mathrm{d} \alpha_{s}} \\
& \mathfrak{R}\left(\widehat{\alpha}_{s} \mid x\right)_{\mathrm{QLF}(J)}=1-\frac{\left\{1 / \Lambda_{J} \sum_{j=0}^{n-r} \Phi_{J}(s) \int_{\alpha_{s}}\left(\alpha_{s}^{\gamma_{s}-2}\left(\alpha_{s}^{2}+4 \alpha_{s}+2\right)^{1 / 2} /\left(1+\alpha_{k}\right)^{r_{s}+m_{s}+1}\right) e^{-\alpha_{s}\left\{\psi_{s}(x)\right\}} \mathrm{d} \alpha_{s}\right\}^{2}}{1 / \Lambda_{J} \sum_{j=0}^{n-r} \Phi_{J}(s) \int_{\alpha_{s}}\left(\alpha_{s}^{\gamma_{s}-3}\left(\alpha_{s}^{2}+4 \alpha_{s}+2\right)^{1 / 2} /\left(1+\alpha_{k}\right)^{r_{s}+m_{s}+1}\right) e^{-\alpha_{s}\left\{\psi_{s}(x)\right\}} \mathrm{d} \alpha_{s}}, \\
& \left(\widehat{\lambda}_{1} \mid x\right)_{\mathrm{QLF}(J)}=\frac{\sum_{j=0}^{n-r} \Phi_{J 1} B\left(v_{1}, v_{2}+v_{3}+2\right)}{\sum_{j=0}^{n-r} \Phi_{J 1} B\left(v_{1}-1, v_{2}+v_{3}+2\right)},
\end{aligned}
$$




$$
\begin{aligned}
& \mathfrak{R}\left(\widehat{\lambda}_{1} \mid x\right)_{Q L F(J)}=1-\frac{\left\{1 / \Lambda_{J} \sum_{j=0}^{n-r} \Phi_{J 1} B\left(v_{1}, v_{2}+v_{3}+2\right)\right\}^{2}}{1 / \Lambda_{J} \sum_{j=0}^{n-r} \Phi_{J 1} B\left(v_{1}-1, v_{2}+v_{3}+2\right)}, \\
& \left(\widehat{\lambda}_{2} \mid x\right)_{Q L F(J)}=\frac{\sum_{j=0}^{n-r} \Phi_{J 2} B\left(v_{2}, v_{1}+v_{3}+2\right)}{\sum_{j=0}^{n-r} \Phi_{J 2} B\left(v_{2}-1, v_{1}+v_{3}+2\right)}, \\
& \mathfrak{R}\left(\hat{\lambda}_{2} \mid x\right)_{Q L F(J)}=1-\frac{\left\{1 / \Lambda_{J} \sum_{j=0}^{n-r} \Phi_{J 2} B\left(v_{2}, v_{1}+v_{3}+2\right)\right\}^{2}}{1 / \Lambda_{J} \sum_{j=0}^{n-r} \Phi_{J 2} B\left(v_{2}-1, v_{1}+v_{3}+2\right)} \\
& \left(\widehat{\alpha}_{s} \mid x\right)_{W L F(J)}=\left\{\frac{1}{\Lambda_{J}} \sum_{j=0}^{n-r} \Phi_{J}(s) \int_{\alpha_{s}} \frac{\alpha_{s}^{\gamma_{s}-2}\left(\alpha_{s}^{2}+4 \alpha_{s}+2\right)^{1 / 2}}{\left(1+\alpha_{k}\right)^{r_{s}+m_{s}+1}} e^{-\alpha_{s}\left\{\psi_{s}(x)\right\}} \mathrm{d} \alpha_{s}\right\}^{-1}, \\
& \mathfrak{R}\left(\widehat{\alpha}_{s} \mid x\right)_{W L F(J)}=\frac{1}{\Lambda_{J}} \sum_{j=0}^{n-r} \Phi_{J}(s) \int_{\alpha_{s}} \frac{\alpha_{s}^{\gamma_{s}}\left(\alpha_{s}^{2}+4 \alpha_{s}+2\right)^{1 / 2}}{\left(1+\alpha_{k}\right)^{r_{s}+m_{s}+1}} e^{-\alpha_{s}\left\{\psi_{s}(x)\right\}} \mathrm{d} \alpha_{s}-\left(\widehat{\alpha}_{s} \mid x\right)_{W L F(J)}, \\
& \left(\widehat{\lambda}_{1} \mid x\right)_{W L F(J)}=\left\{\frac{1}{\Lambda_{J}} \sum_{j=0}^{n-r} \Phi_{J 1} B\left(v_{1}, v_{2}+v_{3}+2\right)\right\}^{-1} \\
& \mathfrak{R}\left(\hat{\lambda}_{1} \mid x\right)_{W L F(J)}=\frac{1}{\Lambda_{J}} \sum_{j=0}^{n-r} \Phi_{J 1} B\left(v_{1}+2, v_{2}+v_{3}+2\right)-\left(\hat{\lambda}_{1} \mid x\right)_{W L F(J)}, \\
& \left(\widehat{\alpha}_{s} \mid x\right)_{P L F(J)}=\left\{\frac{1}{\Lambda_{J}} \sum_{j=0}^{n-r} \Phi_{J}(s) \int_{\alpha_{s}} \frac{\alpha_{s}^{\gamma_{s}+1}\left(\alpha_{s}^{2}+4 \alpha_{s}+2\right)^{1 / 2}}{\left(1+\alpha_{k}\right)^{r_{s}+m_{s}+1}} e^{-\alpha_{s}\left\{\psi_{s}(x)\right\}} \mathrm{d} \alpha_{s}\right\}^{1 / 2}, \\
& \mathfrak{R}\left(\widehat{\alpha}_{s} \mid x\right)_{P L F(J)}=2\left\{\left(\widehat{\alpha}_{s} \mid x\right)_{P L F(J)}-\frac{1}{\Lambda_{J}} \sum_{j=0}^{n-r} \Phi_{J}(s) \int_{\alpha_{s}} \frac{\alpha_{s}^{\gamma_{s}}\left(\alpha_{s}^{2}+4 \alpha_{s}+2\right)^{1 / 2}}{\left(1+\alpha_{k}\right)^{r_{s}+m_{s}+1}} e^{-\alpha_{s}\left\{\psi_{s}(x)\right\}} \mathrm{d} \alpha_{s}\right\}, \\
& \left(\widehat{\lambda}_{1} \mid x\right)_{P L F(J)}=\left\{\frac{1}{\Lambda_{J}} \sum_{j=0}^{n-r} \Phi_{J 1} B\left(v_{1}+3, v_{2}+v_{3}+2\right)\right\}^{1 / 2} \\
& \mathfrak{R}\left(\hat{\lambda}_{1} \mid x\right)_{P L F(J)}=2\left\{\left(\hat{\lambda}_{1} \mid x\right)_{P L F(J)}-\frac{1}{\Lambda_{J}} \sum_{j=0}^{n-r} \Phi_{J 1} B\left(v_{1}+2, v_{2}+v_{3}+2\right)\right\} \\
& \left(\widehat{\lambda}_{2} \mid x\right)_{P L F(J)}=\left\{\frac{1}{\Lambda_{J}} \sum_{j=0}^{n-r} \Phi_{J 2} B\left(v_{2}+3, v_{1}+v_{3}+2\right)\right\}^{1 / 2} \\
& \mathfrak{R}\left(\hat{\lambda}_{1} \mid x\right)_{P L F(J)}=2\left\{\left(\hat{\lambda}_{1} \mid x\right)_{P L F(J)}-\frac{1}{\Lambda_{J}} \sum_{j=0}^{n-r} \Phi_{J 2} B\left(v_{2}+2, v_{1}+v_{3}+2\right)\right\}
\end{aligned}
$$

\section{Simulation Study}

The preceding sections provide a basis to formulate the BEs and PRs for parameter under different loss functions using Bayesian inference. The BEs and PRs have been derived for the loss functions (provided in Table 1) under Type-I censoring scheme. In this section, Monte Carlo simulations have been performed under varying conditions to assess the viability of the BEs derived in the previous section. For the sake of comparative analysis, the PRs for the BEs under 
TABle 2: BEs and PRs for 3-CULD under Scheme-I assuming gamma prior.

\begin{tabular}{|c|c|c|c|c|c|c|c|c|c|c|}
\hline \multirow{2}{*}{$n$} & & & \multicolumn{4}{|c|}{$\gamma_{0}=0.6$} & \multicolumn{4}{|c|}{$\gamma_{0}=0.8$} \\
\hline & & & SELF & QLF & WLF & PLF & SELF & QLF & WLF & PLF \\
\hline \multirow{10}{*}{20} & \multirow{2}{*}{$\widehat{\alpha}_{1}$} & $\mathrm{BE}$ & 0.5027 & 0.5027 & 0.5029 & 0.5018 & 0.5478 & 0.5353 & 0.5495 & 0.5484 \\
\hline & & PR & 0.0763 & 0.0059 & 0.0029 & 0.0011 & 0.0937 & 0.0097 & 0.0071 & 0.0057 \\
\hline & \multirow{2}{*}{$\widehat{\alpha}_{2}$} & $\mathrm{BE}$ & 1.0676 & 1.0471 & 1.0695 & 1.0409 & 1.0718 & 1.0700 & 1.0757 & 1.0749 \\
\hline & & PR & 0.0985 & 0.0047 & 0.0053 & 0.0056 & 0.1058 & 0.0050 & 0.0056 & 0.0057 \\
\hline & \multirow{2}{*}{$\widehat{\alpha}_{3}$} & $\mathrm{BE}$ & 2.0509 & 2.0453 & 2.0449 & 2.0329 & 2.0626 & 2.0659 & 2.0791 & 2.0643 \\
\hline & & PR & 0.0551 & 0.0012 & 0.0028 & 0.0029 & 0.0711 & 0.0014 & 0.0032 & 0.0029 \\
\hline & \multirow{2}{*}{$\hat{\lambda}_{1}$} & $\mathrm{BE}$ & 0.5658 & 0.5735 & 0.5851 & 0.5739 & 0.5715 & 0.5311 & 0.5523 & 0.5804 \\
\hline & & PR & 0.0099 & 0.0209 & 0.0147 & 0.0126 & 0.0098 & 0.0217 & 0.0187 & 0.0168 \\
\hline & \multirow{2}{*}{$\hat{\lambda}_{2}$} & $\mathrm{BE}$ & 0.1792 & 0.1629 & 0.1586 & 0.1857 & 0.1845 & 0.1499 & 0.1815 & 0.1901 \\
\hline & & PR & 0.0161 & 0.2146 & 0.0338 & 0.0325 & 0.0157 & 0.2103 & 0.0857 & 0.0315 \\
\hline \multirow{10}{*}{50} & \multirow{2}{*}{$\widehat{\alpha}_{1}$} & $\mathrm{BE}$ & 0.5003 & 0.5009 & 0.5006 & 0.5 & 0.5236 & 0.5166 & 0.5311 & 0.5241 \\
\hline & & PR & 0.0516 & 0.0003 & 0.0011 & 0.0004 & 0.0773 & 0.0049 & 0.0049 & 0.0038 \\
\hline & \multirow{2}{*}{$\widehat{\alpha}_{2}$} & $\mathrm{BE}$ & 1.0253 & 1.0298 & 1.0214 & 1.0164 & 1.0567 & 1.0475 & 1.0629 & 1.0796 \\
\hline & & PR & 0.0736 & 0.0029 & 0.0021 & 0.0015 & 0.0855 & 0.0047 & 0.0048 & 0.0051 \\
\hline & \multirow{2}{*}{$\widehat{\alpha}_{3}$} & $\mathrm{BE}$ & 2.0334 & 2.0284 & 2.0285 & 2.0163 & 2.0465 & 2.0454 & 2.0555 & 2.0331 \\
\hline & & PR & 0.0241 & 0.0010 & 0.0016 & 0.0006 & 0.0342 & 0.0011 & 0.0028 & 0.0019 \\
\hline & \multirow{2}{*}{$\hat{\lambda}_{1}$} & $\mathrm{BE}$ & 0.5957 & 0.5789 & 0.5892 & 0.5879 & 0.5883 & 0.5762 & 0.5707 & 0.5824 \\
\hline & & PR & 0.0063 & 0.0139 & 0.0076 & 0.0077 & 0.0044 & 0.0144 & 0.0096 & 0.0076 \\
\hline & \multirow{2}{*}{$\hat{\lambda}_{2}$} & $\mathrm{BE}$ & 0.1819 & 0.1701 & 0.1784 & 0.1886 & 0.1897 & 0.1774 & 0.1885 & 0.1957 \\
\hline & & PR & 0.0073 & 0.0996 & 0.0159 & 0.0114 & 0.0082 & 0.0965 & 0.0243 & 0.0114 \\
\hline \multirow{10}{*}{75} & \multirow{2}{*}{$\widehat{\alpha}_{1}$} & $\mathrm{BE}$ & 0.5000 & 0.5000 & 0.5000 & 0.5000 & 0.5102 & 0.5117 & 0.5251 & 0.5113 \\
\hline & & PR & 0.0333 & $1.0 E-5$ & $7.2 E-5$ & $2.2 E-5$ & 0.0411 & 0.0031 & 0.0038 & 0.0029 \\
\hline & \multirow{2}{*}{$\widehat{\alpha}_{2}$} & $\mathrm{BE}$ & 1.0193 & 1.0139 & 1.0103 & 1.0091 & 1.0411 & 1.0447 & 1.0483 & 1.0767 \\
\hline & & PR & 0.0428 & 0.0019 & 0.0016 & 0.0010 & 0.0536 & 0.0044 & 0.0041 & 0.0050 \\
\hline & & $\mathrm{BE}$ & 2.0201 & 2.0197 & 2.0142 & 2.0072 & 2.0248 & 2.0345 & 2.0425 & 2.0387 \\
\hline & $\alpha_{3}$ & PR & 0.0122 & 0.0004 & 0.0011 & 0.0001 & 0.0227 & 0.0009 & 0.0021 & 0.0014 \\
\hline & & $\mathrm{BE}$ & 0.5985 & 0.5951 & 0.5927 & 0.5886 & 0.5891 & 0.5846 & 0.5802 & 0.5879 \\
\hline & $\lambda_{1}$ & PR & 0.0046 & 0.0051 & 0.0032 & 0.0038 & 0.0029 & 0.0069 & 0.0075 & 0.0052 \\
\hline & $\hat{\lambda}_{2}$ & $\mathrm{BE}$ & 0.1836 & 0.1832 & 0.1883 & 0.1904 & 0.1923 & 0.1912 & 0.1901 & 0.1971 \\
\hline & $\Lambda_{2}$ & PR & 0.0042 & 0.0631 & 0.0093 & 0.0099 & 0.0059 & 0.0614 & 0.0198 & 0.0089 \\
\hline & & $\mathrm{BE}$ & 0.5000 & 0.5000 & 0.5000 & 0.5000 & 0.5013 & 0.5015 & 0.5199 & 0.5039 \\
\hline & $\alpha_{1}$ & PR & 0.0186 & $8.2 E-8$ & $1.3 E-5$ & $2.8 E-6$ & 0.0216 & 0.0021 & 0.0021 & 0.0013 \\
\hline & & $\mathrm{BE}$ & 1.0109 & 1.0091 & 1.0079 & 1.0034 & 1.0238 & 1.0224 & 1.0331 & 1.0735 \\
\hline & $\alpha_{2}$ & PR & 0.0129 & 0.0009 & 0.0011 & 0.0002 & 0.0205 & 0.0042 & 0.0032 & 0.0046 \\
\hline & & $\mathrm{BE}$ & 2.0097 & 2.0051 & 2.0067 & 2.0012 & 2.0198 & 2.0234 & 2.0316 & 2.0261 \\
\hline 100 & $\alpha_{3}$ & PR & 0.0074 & 0.0003 & 0.0004 & $6.3 E-5$ & 0.0118 & 0.0006 & 0.0017 & 0.0013 \\
\hline & $\hat{\lambda}$ & $\mathrm{BE}$ & 0.5997 & 0.5958 & 0.6001 & 0.5923 & 0.5957 & 0.5888 & 0.5851 & 0.5909 \\
\hline & $\lambda_{1}$ & PR & 0.0021 & 0.0027 & 0.0024 & 0.0026 & 0.0021 & 0.0052 & 0.0046 & 0.0039 \\
\hline & & $\mathrm{BE}$ & 0.1901 & 0.1915 & 0.1976 & 0.1911 & 0.1973 & 0.1963 & 0.1938 & 0.1986 \\
\hline & $\lambda_{2}$ & PR & 0.0033 & 0.0397 & 0.0087 & 0.0073 & 0.0032 & 0.0428 & 0.0087 & 0.0071 \\
\hline & & $\mathrm{BE}$ & 0.5000 & 0.5000 & 0.5000 & 0.5000 & 0.5001 & 0.5011 & 0.5072 & 0.5011 \\
\hline & $\alpha_{1}$ & PR & 0.0081 & $1.3 E-8$ & $2.2 E-7$ & $8.2 E-8$ & 0.0112 & 0.0012 & 0.0016 & 0.0011 \\
\hline & & $\mathrm{BE}$ & 1.0032 & 1.0069 & 1.0043 & 1.0006 & 1.0096 & 1.0136 & 1.0202 & 1.0553 \\
\hline & $\alpha_{2}$ & PR & 0.0065 & 0.0002 & 0.0009 & $3.6 E-5$ & 0.0098 & 0.0035 & 0.0029 & 0.0032 \\
\hline & & $\mathrm{BE}$ & 2.0016 & 2.0003 & 2.0021 & 2.0005 & 2.0043 & 2.0047 & 2.0118 & 2.0029 \\
\hline 200 & $\alpha_{3}$ & PR & 0.0032 & 0.0002 & 0.0006 & $1.1 E-5$ & 0.0077 & 0.0001 & 0.0012 & 0.0007 \\
\hline & & $\mathrm{BE}$ & 0.6009 & 0.5981 & 0.6009 & 0.5982 & 0.5987 & 0.5905 & 0.5899 & 0.5946 \\
\hline & $\lambda_{1}$ & PR & 0.0005 & 0.0019 & 0.0017 & 0.0007 & 0.0011 & 0.0041 & 0.0038 & 0.0032 \\
\hline & $\hat{\lambda}$ & $\mathrm{BE}$ & 0.1965 & 0.1954 & 0.1983 & 0.1947 & 0.1983 & 0.1969 & 0.1981 & 0.1991 \\
\hline & $\lambda_{2}$ & PR & 0.0029 & 0.0134 & 0.0046 & 0.0038 & 0.0013 & 0.0189 & 0.0046 & 0.0039 \\
\hline
\end{tabular}

different circumstances are compared. All the simulations are performed using a routine in Mathematica 10.0. For comparative analysis, two simulation schemes along with two test termination times $\gamma_{0}=\{0.6,0.9\}$ were used as follows:
Scheme-I: $\alpha_{1}=0.5, \alpha_{2}=1, \alpha_{3}=2, \lambda_{1}=0.6, \lambda_{2}=0.2$

Scheme-II: $\alpha_{1}=0.5, \alpha_{2}=1, \alpha_{3}=2, \lambda_{1}=0.4, \lambda_{2}=0.3$

The samples of 3-CULD mixtures are generated, using Mathematica, as follows: 


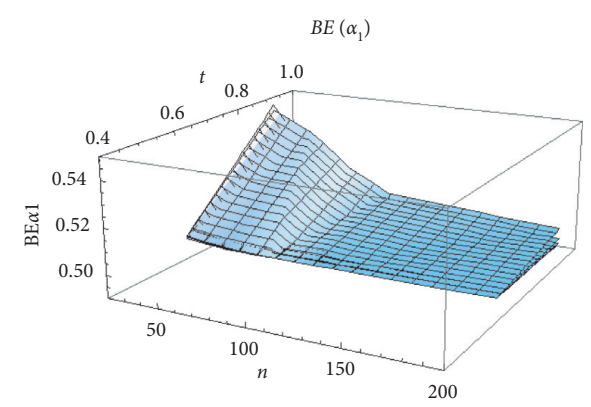

(a)

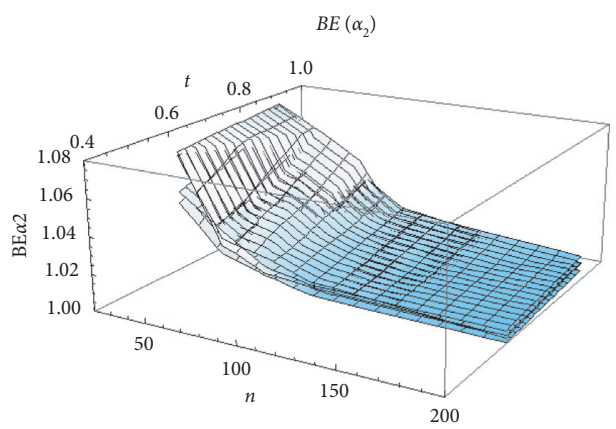

(c)

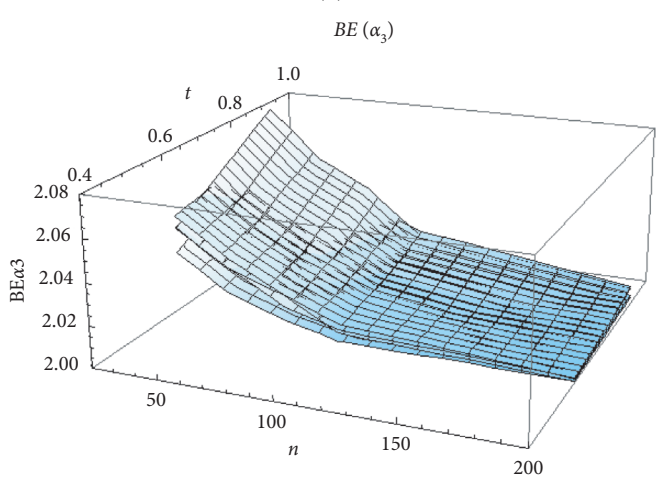

(e)

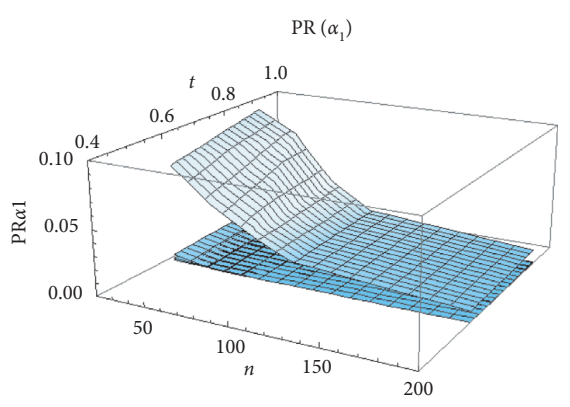

(b)

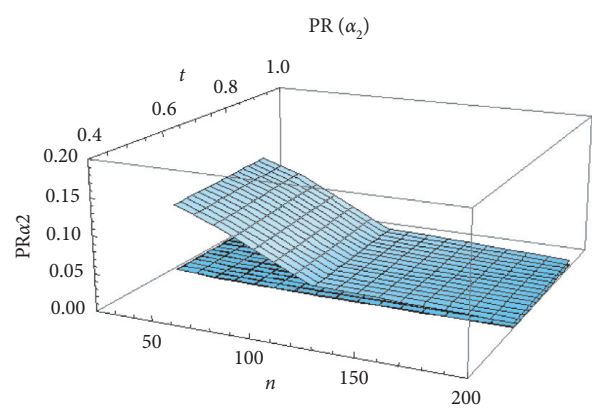

(d)

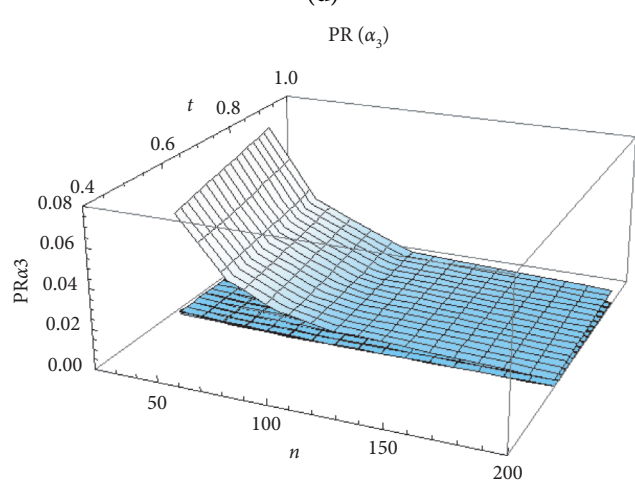

(f)

Figure 1: 3D plots for the BEs and PRs under Jeffreys prior. (a) $B E\left(\alpha_{1}\right)$, (b) $P R\left(\alpha_{1}\right)$, (c) $B E\left(\alpha_{2}\right)$, (d) $P R\left(\alpha_{2}\right),(\mathrm{e}) B E\left(\alpha_{3}\right)$, and (f) $P R\left(\alpha_{3}\right)$.

TABLE 3: BEs and PRs under gamma prior.

\begin{tabular}{|c|c|c|c|c|c|}
\hline \multicolumn{2}{|c|}{ Loss Functions } & \multirow{2}{*}{$\begin{array}{c}\text { SELF } \\
1.56\end{array}$} & \multirow{2}{*}{$\begin{array}{c}\text { QLF } \\
1.56\end{array}$} & \multirow{2}{*}{$\begin{array}{c}\text { WLF } \\
1.56\end{array}$} & \multirow{2}{*}{$\begin{array}{c}\text { PLF } \\
1.56\end{array}$} \\
\hline & $\mathrm{BE}$ & & & & \\
\hline$\alpha_{1}$ & PR & $4.81 E-7$ & $1.74 E-7$ & $2.89 E-7$ & $3.08 E-7$ \\
\hline \multirow{2}{*}{$\widehat{\alpha}_{2}$} & $\mathrm{BE}$ & 1.47 & 1.47 & 1.47 & 1.47 \\
\hline & PR & $7.42 E-9$ & $3.01 E-9$ & $4.73 E-9$ & $5.05 E-9$ \\
\hline \multirow{2}{*}{$\widehat{\alpha}_{3}$} & $\mathrm{BE}$ & 1.48 & 1.48 & 1.48 & 1.48 \\
\hline & PR & $5.13 E-10$ & $2.05 E-10$ & $3.25 E-10$ & $3.47 E-10$ \\
\hline \multirow{2}{*}{$\hat{\lambda}_{1}$} & $\mathrm{BE}$ & 0.3954 & 0.3561 & 0.3759 & 0.4032 \\
\hline & PR & 0.0069 & 0.0196 & 0.0186 & 0.0173 \\
\hline \multirow{2}{*}{$\hat{\lambda}_{2}$} & $\mathrm{BE}$ & 0.2351 & 0.1819 & 0.2094 & 0.2468 \\
\hline & PR & 0.0056 & 0.0911 & 0.0911 & 0.0234 \\
\hline
\end{tabular}

(i) Generate $n \lambda_{1}$ random observations from the first component density.

(ii) Generate $n \lambda_{2}$ random observations from the second component density.

(iii) Generate remaining $n\left(1-\lambda_{1}-\lambda_{2}\right)$ random observations from the third component density. (iv) A sample censored at the fixed test termination time is selected. The observations which are greater than $\gamma_{0}$ are identified as censored ones.

(v) Repeat steps (i) to (iv) to generate 1000 samples of sizes $n=(20,50,75,100,200)$ assuming different parametric values fixed as $\left(\alpha_{1}, \alpha_{2}, \alpha_{3}, \lambda_{1}, \lambda_{2}\right)$ $=\{(0.5,1,2,0.6,0.2),(0.5,1,2,0.3,0.4)\}$. 
TABle 4: BEs and PRs under uniform prior.

\begin{tabular}{|c|c|c|c|c|c|}
\hline \multicolumn{2}{|c|}{ Loss functions } & SELF & QLF & WLF & PLF \\
\hline \multirow{2}{*}{$\widehat{\alpha}_{1}$} & $\mathrm{BE}$ & 1.56 & 1.56 & 1.56 & 1.56 \\
\hline & PR & $4.76 E-7$ & $1.73 E-7$ & $2.87 E-7$ & $3.05 E-7$ \\
\hline \multirow{2}{*}{$\widehat{\alpha}_{2}$} & $\mathrm{BE}$ & 1.47 & 1.47 & 1.47 & 1.47 \\
\hline & PR & $8.04 E-9$ & $3.26 E-9$ & $5.12 E-9$ & $5.47 E-9$ \\
\hline \multirow{2}{*}{$\widehat{\alpha}_{3}$} & $\mathrm{BE}$ & 1.48 & 1.48 & 1.48 & 1.48 \\
\hline & PR & $5.89 E-10$ & $2.36 E-10$ & $3.72 E-10$ & $3.98 E-10$ \\
\hline \multirow{2}{*}{$\hat{\lambda}_{1}$} & $\mathrm{BE}$ & 0.3985 & 0.3598 & 0.3793 & 0.3985 \\
\hline & PR & 0.0072 & 0.00237 & 0.0191 & 0.0178 \\
\hline \multirow{2}{*}{$\hat{\lambda}_{2}$} & $\mathrm{BE}$ & 0.2706 & 0.2179 & 0.2452 & 0.2706 \\
\hline & PR & 0.0064 & 0.1081 & 0.0254 & 0.0232 \\
\hline
\end{tabular}

TABLE 5: BEs and PRs under Jeffreys prior.

\begin{tabular}{|c|c|c|c|c|c|}
\hline \multicolumn{2}{|c|}{ Loss functions } & SELF & QLF & WLF & PLF \\
\hline \multirow{2}{*}{$\widehat{\alpha}_{1}$} & $\mathrm{BE}$ & 1.56 & 1.56 & 1.56 & 1.56 \\
\hline & PR & $4.45 E-7$ & $1.61 E-7$ & $2.96 E-7$ & $2.85 E-7$ \\
\hline \multirow[b]{2}{*}{$\widehat{\alpha}_{2}$} & $\mathrm{BE}$ & 1.47 & 1.47 & 1.47 & 1.47 \\
\hline & PR & $7.49 E-9$ & $3.04 E-9$ & $5.30 E-9$ & $5.09 E-E-9$ \\
\hline \multirow{2}{*}{$\widehat{\alpha}_{3}$} & $\mathrm{BE}$ & 1.48 & 1.48 & 1.48 & 1.48 \\
\hline & PR & $5.48 E-10$ & $2.19 E-10$ & $3.85 E-10$ & $3.70 E-10$ \\
\hline \multirow{2}{*}{$\hat{\lambda}_{1}$} & $\mathrm{BE}$ & 0.3985 & 0.3598 & 0.3793 & 0.3985 \\
\hline & PR & 0.0072 & 0.00237 & 0.0191 & 0.0178 \\
\hline \multirow{2}{*}{$\hat{\lambda}_{2}$} & $\mathrm{BE}$ & 0.2706 & 0.2179 & 0.2452 & 0.2706 \\
\hline & PR & 0.0064 & 0.1081 & 0.0254 & 0.0232 \\
\hline
\end{tabular}

(vi) Calculate the Bayes estimates and posterior risks of the parameters from the samples generated in step (v).

Table 2 provides the simulation results for 3-CULD under Scheme-I assuming the gamma priors and said loss functions.

The $3 \mathrm{D}$ plots for the BEs and their respective PRs under simulation Scheme-I assuming Jeffreys prior are provided in Figure 1.

The simulation results from Scheme-I reveal that the gamma prior out performs all the other priors in term of minimizing the PRs while Jeffreys prior performs better among the noninformative priors. Among various loss functions considered, the loss functions performance w.r.t their respective PRs, from best to least, is as follows: $\mathrm{PLF}<\mathrm{WLF}<\mathrm{QLF}<\mathrm{SELF}$. PRs are smaller for smaller test termination time, i.e., $\gamma_{0}=0.6$, as compared to those for $\gamma_{0}=$ 0.9 and the estimates converge more rapidly for $\gamma_{0}=0.6$ than those for $\gamma_{0}=0.9$. Furthermore, as the sample sizes increase, the PRs tend to decrease.

The results obtained under Scheme-II conform to the inferences drawn from the results obtained under Scheme-I.
The comparative analysis of the two schemes depicts that the BEs of the component parameters with larger mixing proportions converge more rapidly than those with smaller mixing proportions.

\section{Real Life Application}

For illustrative purpose, real life flood data from Abdel-Latif and Yacoub [23] have been analyzed in this section. El-Deen et al. [24] transformed the data to the interval $[0,1]$ by dividing the capacities over the capacity of the reservoir and fitted Kumaraswamy distribution to the data. However, we have assumed that the data can be categorized into three subpopulations belonging to unit Lindley distribution.

The data set consists of annual water level behind the high dam during the flood time from 1980 to 2010. The highest water level of the dam is 182 meters (m) above the mean sea level. The transformed data used for the analysis are

$\begin{array}{llllllllll}0.9402 & 0.9360 & 0.9113 & 0.8989 & 0.8592 & 0.8639 & 0.8497 & 0.8335 & 0.9033 & 0.8998 \\ 0.8928 & 0.9009 & 0.9200 & 0.9320 & 0.9469 & 0.9492 & 0.9641 & 0.9601 & 0.9601 & 0.9658 \\ 0.9662 & 0.9653 & 0.9623 & 0.9453 & 0.9318 & 0.9266 & 0.9528 & 0.9604 & 0.9521 & 0.9329\end{array}$

Assuming the mixing proportions, $\lambda_{1}=0.4, \lambda_{2}=0.3, \lambda_{3}=0.3$, we categorize the data into three underlying populations. The Kolmogorov-Smirnov test shows that each of the three subpopulations follows unit Lindley with $p$ values 0.4740 , 0.1273 , and 0.4740 , respectively. For the threshold (test termination time) $\gamma_{0}=0.965$, the data are summarized as follows: 


$$
\begin{aligned}
r & =27, \\
r_{1} & =12, \\
r_{2} & =7, \\
r_{3} & =8, \\
\sum_{k=1}^{r_{1}} \frac{x_{1 k}}{1-x_{1 k}} & =108.421, \\
\sum_{k=1}^{r_{2}} \frac{x_{2 k}}{1-x_{2 k}} & =136.829, \\
\sum_{k=1}^{r_{3}} \frac{x_{3 k}}{1-x_{3 k}} & =147.438 .
\end{aligned}
$$

Since $n-r=3$, the censoring rate is 10 percent. Thus, the Bayes estimates and their risks using the loss functions for all three priors under studies are presented in Tables 3-5.

The results from the analysis of flood data are compatible with the results obtained under simulation study. The BEs obtained under PLF have the smallest PRs and thus can be considered as the most efficient BEs among the other loss functions. Among the different priors, gamma prior has the lowest PRs associated with the BEs as compared to the noninformative priors.

\section{Conclusion}

This paper investigates the 3-CULD mixture in context of flood data under Bayesian paradigm. The right censored 3CULD model is formulated assuming informative and noninformative priors. The expressions for BEs and their PRs are derived assuming different loss functions and the efficiency of these BEs were analyzed w.r.t their PRs under two contrasting simulation schemes. The simulation results reinforce the popular opinion that the informative prior always provides better results in Bayesian analysis. It can be seen from the simulation study as well as real life data analysis that the BEs obtained under gamma prior performed better, in terms of smaller risks, than those obtained under noninformative priors. Among the noninformative priors, Jeffreys prior provides more efficient estimates than those obtained under uniform prior as the BEs obtained under the Jeffreys prior have smaller risks. The simulation results further indicate that the asymmetric loss functions yield more efficient estimates (smaller risks) than the symmetric loss function. It is also noted that BEs under QLF perform better when estimating the component parameters and SELF provides BEs with minimum risks for estimating mixture proportions. However, for estimating the overall mixture, BEs assuming PLF outperform the BEs under WLF, QLF, and SELF. It is also speculated that the efficiency of BEs is indirectly proportional to the test termination time and directly proportional to sample sizes and censoring rate. Finally, the analysis is extended to study the real life application to flood data; the proposed model is able to model the bulk data for below the threshold value quiet proficiently. Moreover, the real data analysis endorses the inferences drawn under the simulation study.

\section{Appendix}

\section{A.1. Likelihood Function}

The derivation of likelihood function in (3) is

$$
\begin{aligned}
L(\Omega \mid x) \propto & \prod_{i=1}^{r_{1}} \lambda_{1} \frac{\alpha_{1}}{1+\alpha_{1}}(1-x)^{-3} e^{-\left(\alpha_{1} x / 1-x\right)} \prod_{i=1}^{r_{2}} \lambda_{2} \frac{\alpha_{2}}{1+\alpha_{2}}(1-x)^{-3} e^{-\left(\alpha_{2} x / 1-x\right)} \prod_{i=1}^{r_{3}} \lambda_{3} \frac{\alpha_{3}}{1+\alpha_{3}}(1-x)^{-3} e^{-\left(\alpha_{3} x / 1-x\right)} \\
& \left\{\sum_{l=1}^{3} \lambda_{l}\left(1+\frac{\alpha_{l} \gamma_{0}}{\left(1+\alpha_{l}\right)\left(1-\gamma_{0}\right)}\right) e^{-\left(\alpha_{l} \gamma_{0} / 1-\gamma_{0}\right)}\right\}^{n-r}, \\
L(\Omega \mid x) \propto & \lambda_{1}^{r 1+n-r-j} \lambda_{2}^{r 2+j-k 1} \lambda_{3}^{r 3+j}\left\{\prod_{l=1}^{3} \frac{\alpha_{l}^{2 r l}}{\left(1+\alpha_{l}\right)^{r l}}\right\} e^{-\alpha_{1}\left(\sum_{k=1}^{r 1}\left(x_{l k} / 1-x_{l k}\right)\right)-\alpha_{2}\left(\sum_{k=1}^{r 2}\left(x_{l k} / 1-x_{l k}\right)\right)-\alpha_{3}\left(\sum_{k=1}^{r 3}\left(x_{l k} / 1-x_{l k}\right)\right)} \\
& \left\{\sum_{l=1}^{3} \lambda_{l}\left(1+\frac{\alpha_{l} \gamma_{0}}{\left(1+\alpha_{l}\right)\left(1-\gamma_{0}\right)}\right) e^{-\left(\alpha_{l} \gamma_{0} / 1-\gamma_{0}\right)}\right\}^{n-r}, \\
L(\Omega \mid x) \propto & \sum_{j=0}^{n-r} \sum_{m=0}^{j}\left(\begin{array}{c}
n-r \\
j
\end{array}\right)\left(\begin{array}{c}
j \\
m
\end{array}\right) \lambda_{1}^{r 1+n-r-j} \lambda_{2}^{r 2+j-m} \lambda_{3}^{r 3+j} \\
& \left\{\prod_{k=1}^{3} \frac{\alpha_{k}^{2 r k}}{\left(1+\alpha_{k}\right)^{r_{k}}}\right\} e^{-\alpha_{1}}\left\{\sum_{i=1}^{r 1}\left(x_{l i} / 1-x_{l i}\right)+\left((n-r-j) \gamma_{0} / 1-\gamma_{0}\right)\right\}-\alpha_{2}\left\{\sum_{i=1}^{r 2}\left(x_{l i} / 1-x_{l i}\right)+\left((j-k) \gamma_{0} / 1-\gamma_{0}\right)\right\}-\alpha_{3}\left\{\sum_{i=1}^{r 3}\left(x_{l i} / 1-x_{l i}\right)+\left(j \gamma_{0} / 1-\gamma_{0}\right)\right\} \\
& \left\{1+\frac{\alpha_{1} \gamma_{0}}{\left(1+\alpha_{l}\right)\left(1-\gamma_{0}\right)}\right\}^{n-r-j}\left\{1+\frac{\alpha_{2} \gamma_{0}}{\left(1+\alpha_{2}\right)\left(1-\gamma_{0}\right)}\right\}^{j-m}\left\{1+\frac{\alpha_{3} \gamma_{0}}{\left(1+\alpha_{l}\right)\left(1-\gamma_{0}\right)}\right\}^{j},
\end{aligned}
$$


$L(\Omega \mid x) \propto \sum_{j=0}^{n-r} \sum_{m=0}^{j} \sum_{m_{1}=0}^{n-r-j} \sum_{m_{2}=0}^{j-m_{1}} \sum_{m_{3}=0}^{m_{1}}\left(\begin{array}{c}n-r \\ j\end{array}\right)\left(\begin{array}{c}j \\ m\end{array}\right)\left(\begin{array}{c}n-r-j \\ m_{1}\end{array}\right)\left(\begin{array}{c}j-m \\ m_{2}\end{array}\right)\left(\begin{array}{c}m \\ m_{3}\end{array}\right) \lambda_{1}^{r 1+n-r-j+m_{1}} \lambda_{2}^{r 2+j-k+m_{2}} \lambda_{3}^{r 3+j+m_{3}}\left(\frac{\gamma_{0}}{1-\gamma_{0}}\right)^{m_{1}+m_{2}+m_{3}}$ $\left\{\prod_{k=1}^{3} \frac{\alpha_{k}^{2 r_{k}+m_{k}}}{\left(1+\alpha_{l}\right)^{r_{k}+m_{k}}}\right\} e^{-\alpha_{1}\left\{\sum_{k=1}^{r 1}\left(x_{l k} / 1-x_{l k}\right)+\left((n-r-j) \gamma_{0} / 1-\gamma_{0}\right)\right\}-\alpha_{2}\left\{\sum_{k=1}^{r 2}\left(x_{l k} / 1-x_{l k}\right)+\left((j-k) \gamma_{0} / 1-\gamma_{0}\right)\right\}-\alpha_{3}\left\{\sum_{k=1}^{r 3}\left(x_{l k} / 1-x_{l k}\right)+\left(j \gamma_{0} / 1-\gamma_{0}\right)\right\},}$

$L(\Omega \mid x) \propto \sum_{j=0}^{n-r} \sum_{m=0}^{j} \sum_{m_{1}=0}^{n-r-j} \sum_{m_{2}=0}^{j-m_{1}} \sum_{m_{3}=0}^{m_{1}} \Phi \lambda_{1}^{r 1+n-r-j} \lambda_{2}^{r 2+j-m}\left(1-\lambda_{1}\right)^{r_{3}+j} \prod_{k=1}^{3} \frac{\alpha_{k}^{2 r_{k}+m_{k}}}{\left(1+\alpha_{k}\right)^{r_{k}+m_{k}}} e^{-\alpha_{k}\left\{\psi_{k}(x)\right\}}$,

where

$$
\begin{aligned}
\Omega & =\left\{\alpha_{1}, \alpha_{2}, \alpha_{3}, \lambda_{1}, \lambda_{2}\right\}, \\
\Phi & =\left(\begin{array}{c}
n-r \\
j
\end{array}\right)\left(\begin{array}{c}
j \\
m
\end{array}\right)\left(\begin{array}{c}
n-r-j \\
m_{1}
\end{array}\right)\left(\begin{array}{c}
j-m \\
m_{2}
\end{array}\right)\left(\begin{array}{c}
m \\
m_{3}
\end{array}\right)\left(\frac{\gamma_{0}}{1-\gamma_{0}}\right)^{m_{1}+m_{2}+m_{3}}, \\
\psi_{1}(x) & =\sum_{1}^{r_{1}} \frac{x}{1-x}+\frac{(n-r-j) \gamma_{0}}{1-\gamma_{0}}, \\
\psi_{2}(x) & =\sum_{1}^{r_{2}} \frac{x}{1-x}+\frac{(j-m) \gamma_{0}}{1-\gamma_{0}}, \\
\psi_{3}(x) & =\sum_{1}^{r_{3}} \frac{x}{1-x}+\frac{j \gamma_{0}}{1-\gamma_{0}} .
\end{aligned}
$$

\section{A.2. Derivation of Posterior Distribution and Marginal Posterior Distributions}

A.2.1. Assuming Informative Prior. The derivation of the posterior distribution and marginal posterior under informative prior is as follows:

The informative priors assumed for component parameters $\alpha_{l}$ and mixing parameters $\left(\lambda_{1}, \lambda_{2}\right)$ are gamma $\left(a_{l}, b_{l}\right)$ and Dirichlet $(a, b, c)$, respectively. The joint prior distribution is thus formulated as

$$
\pi_{g}(\Omega) \propto \lambda_{1}^{a-1} \lambda_{2}^{b-1} \lambda_{3}^{c-1} \alpha_{1}^{a_{1}-1} e^{-b_{1} \alpha_{1}} \alpha_{2}^{a_{2}-1} e^{-b_{2} \alpha_{2}} \alpha_{3}^{a_{3}-1} e^{-b_{3} \alpha_{3}} .
$$

The posterior distribution is thus derived as

$$
\begin{aligned}
& p_{g}(\Omega \mid x)=\frac{\pi_{g}(\Omega) L(\Omega \mid x)}{\iiint_{\Omega} \pi_{g}(\Omega) L(\Omega \mid x) \mathrm{d} \Omega}, \\
& p_{g}(\Omega \mid x)=\frac{\sum_{j=0}^{n-r} \sum_{m=0}^{j} \sum_{m_{1}=0}^{n-r-j} \sum_{m_{2}=0}^{j-m_{1}} \sum_{m_{3}=0}^{m_{1}} \Phi \lambda_{1}^{r_{1}+n-r-j+a-1} \lambda_{2}^{r_{2}+j-m+b-1} \lambda_{3}^{r_{3}+j+c-1} \prod_{k=1}^{3}\left(\alpha_{k}^{2 r_{k}+m_{k}+a_{k}-1} /\left(1+\alpha_{k}\right)^{r_{k}+m_{k}}\right) e^{-\alpha_{k}\left\{\psi_{k}(x)+b_{k}\right\}}}{\sum_{j=0}^{n-r} \sum_{m=0}^{j} \sum_{m_{1}=0}^{n-r-j} \sum_{m_{2}=0}^{j-m_{1}} \sum_{m_{3}=0}^{m_{1}} \Phi \iint_{\Omega} \lambda_{1}^{r_{1}+n-r-j+a-1} \lambda_{2}^{r_{2}+j-m+b-1} \lambda_{3}^{r_{3}+j+c-1} \prod_{k=1}^{3}\left(\alpha_{k}^{2 r_{k}+m_{k}+a_{k}-1} /\left(1+\alpha_{k}\right)^{r_{k}+m_{k}}\right) e^{-\alpha_{k}\left\{\psi_{k}(x)+b_{k}\right\}} \mathrm{d} \Omega}, \\
& p_{g}(\Omega \mid x)=\frac{1}{\Lambda_{g}} \sum_{j=0}^{n-r} \sum_{m=0}^{j} \sum_{m_{1}=0}^{n-r-j} \sum_{m_{2}=0}^{j-m_{1}} \sum_{m_{3}=0}^{m_{1}} \Phi \lambda_{1}^{v_{g 1}-1} \lambda_{2}^{v_{g 2}-1} \lambda_{3}^{v_{g 3}-1} \prod_{k=1}^{3} \frac{\alpha_{k}^{\gamma_{g k}-1}}{\left(1+\alpha_{k}\right)^{r_{k}+m_{k}}} e^{-\alpha_{k}\left\{\psi_{k}(x)+b_{k}\right\}},
\end{aligned}
$$

where

$$
\begin{aligned}
\Lambda_{g}= & \sum_{j=0}^{n-r} \sum_{m=0}^{j} \sum_{m_{1}=0}^{n-r-j} \sum_{m_{2}=0}^{j-m_{1}} \sum_{m_{3}=0}^{m_{1}} \Phi B\left(v_{g 1}, v_{g 2}+v_{g 3}\right) B \\
& \left(v_{g 2}, v_{g 1}+v_{g 3}\right) \prod_{k=1}^{3} \int_{\alpha_{k}} \frac{\alpha_{k}^{\gamma_{g k}-1}}{\left(1+\alpha_{k}\right)^{r_{k}+m_{k}}} e^{-\alpha_{k} \delta_{g k}} \mathrm{~d} \alpha_{k},
\end{aligned}
$$




$$
\begin{aligned}
& v_{g 1}=r_{1}+n-r-j+a, \\
& v_{g 2}=r_{2}+j-m+b, \\
& v_{g 3}=r_{3}+j+c, \\
& \gamma_{g s}=2 r_{s}+m_{s}+a_{s}, \\
& \delta_{g s}=\psi_{s}(x)+b_{s},
\end{aligned}
$$

and the derivation of the respective marginal posterior densities is

$$
\begin{aligned}
& p_{g}\left(\alpha_{s} \mid x\right)=\frac{1}{\Lambda_{g}} \sum_{j=0}^{n-r} \sum_{m=0}^{j} \sum_{m_{1}=0}^{n-r-j} \sum_{m_{2}=0}^{j-m_{1}} \sum_{m_{3}=0}^{m_{1}} \Phi \frac{\alpha_{s}^{\gamma_{g s}-1}}{\left(1+\alpha_{s}\right)^{r_{s}+m_{k s}}} e^{-\alpha_{s}\left\{\psi_{s}(x)+b_{s}\right\}} \int_{\lambda_{1}=0}^{1} \int_{\lambda_{2}=0}^{1} \lambda_{1}^{v_{g 1}-1} \lambda_{2}^{v_{g 2}-1} \lambda_{3}^{v_{g 3}-1} \prod_{\substack{k=1 \\
k \neq s}}^{3} \int_{\alpha_{k}=0}^{\infty} \\
& \frac{\alpha_{k}^{\gamma_{g k}-1}}{\left(1+\alpha_{k}\right)^{r_{k}+m_{k}}} e^{-\alpha_{k}\left\{\psi_{k}(x)+b_{k}\right\}} \mathrm{d} \alpha_{k} \mathrm{~d} \lambda_{2} \mathrm{~d} \lambda_{1} \\
& p_{g}\left(\alpha_{s} \mid x\right)=\frac{1}{\Lambda_{g}} \sum_{j=0}^{n-r} \sum_{m=0}^{j} \sum_{m_{1}=0}^{n-r-j} \sum_{m_{2}=0}^{j-m_{1}} \sum_{m_{3}=0}^{m_{1}} \Phi B\left(v_{g 1}, v_{g 2}+v_{g 3}\right) B\left(v_{g 2}, v_{g 1}+v_{g 3}\right) \prod_{\substack{k=1 \\
k \neq s}}^{3} \int_{\alpha_{k}} \frac{\alpha_{k}^{\gamma_{g k}-1}}{\left(1+\alpha_{k}\right)^{r_{k}+m_{k}}} e^{-\alpha_{k} \delta_{g k}} \mathrm{~d} \alpha_{k} \frac{\alpha_{s}^{\gamma_{g s}-1}}{\left(1+\alpha_{s}\right)^{r_{s}+m_{s}}} e^{-\alpha_{s} \delta_{g s}} \\
& p_{g}\left(\alpha_{s} \mid x\right)=\frac{1}{\Lambda_{g}} \sum_{j=0}^{n-r} \Phi_{g}(s) \frac{\alpha_{s}^{\gamma_{g s}-1}}{\left(1+\alpha_{s}\right)^{r_{s}+m_{s}}} e^{-\alpha_{s} \delta_{g s}} \\
& p_{g}\left(\lambda_{1} \mid x\right)=\frac{1}{\Lambda_{g}} \sum_{j=0}^{n-r} \sum_{m=0}^{j} \sum_{m_{1}=0}^{n-r-j} \sum_{m_{2}=0}^{j-m_{1}} \sum_{m_{3}=0}^{m_{1}} \Phi \lambda_{1}^{v_{g 1}-1} \int_{\lambda_{2}=0}^{1} \lambda_{2}^{v_{g 2}-1} \lambda_{3}^{v_{g 3}-1} \prod_{k=1}^{3} \int_{\alpha_{k}=0}^{\infty} \frac{\alpha_{k}^{\gamma_{g s}-1}}{\left(1+\alpha_{k}\right)^{r_{k}+m_{k}}} e^{-\alpha_{k}\left\{\psi_{k}(x)+b_{k}\right\}} \mathrm{d} \alpha_{k} \mathrm{~d} \lambda_{2} \\
& p_{g}\left(\lambda_{1} \mid x\right)=\frac{1}{\Lambda_{g}} \sum_{j=0}^{n-r} \sum_{m=0}^{j} \sum_{m_{1}=0}^{n-r-j} \sum_{m_{2}=0}^{j-m_{1}} \sum_{m_{3}=0}^{m_{1}} \Phi B\left(v_{g 2}, v_{g 1}+v_{g 3}\right) \prod_{\substack{k=1 \\
k \neq s}}^{3} \int_{\alpha_{k}} \frac{\alpha_{k}^{\gamma_{g k}-1}}{\left(1+\alpha_{k}\right)^{r_{k}+m_{k}}} e^{-\alpha_{k} \delta_{g k}} \mathrm{~d} \alpha_{k} \lambda_{1}^{v_{g 1}-1}\left(1-\lambda_{2}\right)^{v_{g 2}+v_{g 3}-1} \\
& p_{g}\left(\lambda_{1} \mid x\right)=\frac{1}{\Lambda_{g}} \sum_{j=0}^{n-r} \Phi_{g 1} \lambda_{1}^{v_{g 1}-1}\left(1-\lambda_{1}\right)^{v_{g 2}+v_{g 3}-1} \\
& p_{g}\left(\lambda_{2} \mid x\right)=\frac{1}{\Lambda_{g}} \sum_{j=0}^{n-r} \sum_{m=0}^{j} \sum_{m_{1}=0}^{n-r-j} \sum_{m_{2}=0}^{j-m_{1}} \sum_{m_{3}=0}^{m_{1}} \Phi \lambda_{2}^{v_{g 2}-1} \int_{\lambda_{1}=0}^{1} \lambda_{1}^{v_{g 1}-1} \lambda_{3}^{v_{g 3}-1} \prod_{k=1}^{3} \int_{\alpha_{k}=0}^{\infty} \frac{\alpha_{k}^{2 r_{k}+m_{k}+a_{k}-1}}{\left(1+\alpha_{k}\right)^{r_{k}+m_{k}}} e^{-\alpha_{k}\left\{\psi_{k}(x)+b_{k}\right\}} \mathrm{d} \alpha_{k} \mathrm{~d} \lambda_{1}, \\
& p_{g}\left(\lambda_{2} \mid x\right)=\frac{1}{\Lambda_{g}} \sum_{j=0}^{n-r} \sum_{m=0}^{j} \sum_{m_{1}=0}^{n-r-j} \sum_{m_{2}=0}^{j-m_{1}} \sum_{m_{3}=0}^{m_{1}} \Phi B\left(v_{g 1}, v_{g 2}+v_{g 3}\right) \prod_{\substack{k=1 \\
k \neq s}}^{3} \int_{\alpha_{k}} \frac{\alpha_{k}^{\gamma_{g k}-1}}{\left(1+\alpha_{k}\right)^{r_{k}+m_{k}}} e^{-\alpha_{k} \delta_{g k}} \mathrm{~d} \alpha_{k} \lambda_{2}^{v_{g 2}-1}\left(1-\lambda_{2}\right)^{v_{g 1}+v_{g 3}-1} \\
& p_{g}\left(\lambda_{2} \mid x\right)=\frac{1}{\Lambda_{g}} \sum_{j=0}^{n-r} \Phi_{g 2} \lambda_{2}^{v_{g 2}-1}\left(1-\lambda_{2}\right)^{v_{g 1}+v_{g 3}-1}
\end{aligned}
$$


where $s=1,2,3$,

$$
\begin{aligned}
\Phi_{g}(s) & =\sum_{m=0}^{j} \sum_{m_{1}=0}^{n-r-j} \sum_{m_{2}=0}^{j-m_{1}} \sum_{m_{3}=0}^{m_{1}} \Phi B\left(v_{g 1}, v_{g 2}+v_{g 3}\right) B\left(v_{g 2}, v_{g 1}+v_{g 3}\right) \prod_{\substack{k=1 \\
k \neq s}}^{3} \int_{\alpha_{k}} \frac{\alpha_{k}^{\gamma_{g k}-1}}{\left(1+\alpha_{k}\right)^{r_{k}+m_{k}}} e^{-\alpha_{k} \delta_{g k}} \mathrm{~d} \alpha_{k}, \\
\Phi_{g 1} & =\sum_{m=0}^{j} \sum_{m_{1}=0}^{n-r-j} \sum_{m_{2}=0}^{j-m_{1}} \sum_{m_{3}=0}^{m_{1}} \Phi B\left(v_{g 2}, v_{g 1}+v_{g 3}\right) \prod_{\substack{k=1 \\
k \neq s}}^{3} \int_{\alpha_{k}} \frac{\alpha_{k}^{\gamma_{g k}-1}}{\left(1+\alpha_{k}\right)^{r_{k}+m_{k}}} e^{-\alpha_{k} \delta_{g k}} \mathrm{~d} \alpha_{k} . \\
\Phi_{g 2} & =\sum_{m=0}^{j} \sum_{m_{1}=0}^{n-r-j} \sum_{m_{2}=0}^{j-m_{1}} \sum_{m_{3}=0}^{m_{1}} \Phi B\left(v_{g 1}, v_{g 2}+v_{g 3}\right) \prod_{\substack{k=1 \\
k \neq s}}^{3} \int_{\alpha_{k}} \frac{\alpha_{k}^{\gamma_{g k}-1}}{\left(1+\alpha_{k}\right)^{r_{k}+m_{k}}} e^{-\alpha_{k} \delta_{g k}} \mathrm{~d} \alpha_{k} .
\end{aligned}
$$

$$
\pi_{u}(\Omega) \propto 1
$$

A.2.2. Assuming Uniform Prior. Assuming uniform prior (UP), the joint prior is
The joint posterior distribution assuming UP is

$$
\begin{aligned}
& p_{u}(\Omega \mid x)=\frac{\pi_{u}(\Omega) L(\Omega \mid x)}{\iiint_{\Omega} \pi_{u}(\Omega) L(\Omega \mid x) \mathrm{d} \Omega}, \\
& p_{u}(\Omega \mid x)=\frac{\sum_{j=0}^{n-r} \sum_{m=0}^{j} \sum_{m_{1}=0}^{n-r-j} \sum_{m_{2}=0}^{j-m_{1}} \sum_{m_{3}=0}^{m_{1}} \Phi \lambda_{1}^{r_{1}+n-r-j} \lambda_{2}^{r_{2}+j-m} \lambda_{3}^{r_{3}+j} \prod_{k=1}^{2}\left(\alpha_{k}^{2 r_{k}+m_{k}} /\left(1+\alpha_{k}\right)^{r_{k}+m_{k}}\right) e^{-\alpha_{k}\left\{\psi_{k}(x)\right\}}}{\sum_{j=0}^{n-r} \sum_{m=0}^{j} \sum_{m_{1}=0}^{n-r-j} \sum_{m_{2}=0}^{j-m_{1}} \sum_{m_{3}=0}^{m_{1}} \Phi \iiint_{\Omega} \lambda_{1}^{r_{1}+n-r-j} \lambda_{2}^{r_{2}+j-m} \lambda_{3}^{r_{3}+j} \prod_{k=1}^{4}\left(\alpha_{k}^{2 r_{k}+m_{k}} /\left(1+\alpha_{k}\right)^{r_{k}+m_{k}}\right) e^{-\alpha_{k}\left\{\psi_{k}(x)\right\}} \mathrm{d} \Omega}, \\
& p_{u}(\Omega \mid x)=\frac{1}{\Lambda_{u}} \sum_{j=0}^{n-r} \sum_{m=0}^{j} \sum_{m_{1}=0}^{n-r-j} \sum_{m_{2}=0}^{j-m_{1}} \sum_{m_{3}=0}^{m_{1}} \Phi \lambda_{1}^{v_{1}} \lambda_{2}^{v_{2}} \lambda_{3}^{v_{3}} \prod_{k=1}^{3} \frac{\alpha_{k}^{\gamma_{k}}}{\left(1+\alpha_{k}\right)^{r_{k}+m_{k}}} e^{-\alpha_{k}\left\{\psi_{k}(x)\right\}},
\end{aligned}
$$

where

$$
\begin{aligned}
\Lambda_{u} & =\sum_{j=0}^{n-r} \sum_{m=0}^{j} \sum_{m_{1}=0}^{n-r-j} \sum_{m_{2}=0}^{j-m_{1}} \sum_{m_{3}=0}^{m_{1}} \Phi B\left(v_{1}+1, v_{2}+v_{3}+2\right) B\left(v_{2}+1, v_{1}+v_{3}+2\right) \prod_{k=1}^{3} \int_{\alpha_{k}} \frac{\alpha_{k}^{\gamma_{k}}}{\left(1+\alpha_{k}\right)^{r_{k}+m_{k}}} e^{-\alpha_{k}\left\{\psi_{k}(x)\right\}} \mathrm{d} \alpha_{k}, \\
v_{1} & =r_{1}+n-r-j \\
v_{2} & =r_{2}+j-m \\
v_{3} & =r_{3}+j \\
\gamma_{s} & =2 r_{s}+m_{s}
\end{aligned}
$$


Also, the respective marginal posterior densities are

$$
\begin{aligned}
& p_{u}\left(\alpha_{s} \mid x\right)=\frac{1}{\Lambda_{u}} \sum_{j=0}^{n-r} \sum_{m=0}^{j} \sum_{m_{1}=0}^{n-r-j} \sum_{m_{2}=0}^{j-m_{1}} \sum_{m_{3}=0}^{m_{1}} \Phi \frac{\alpha_{s}^{2 r_{s}+m_{s}}}{\left(1+\alpha_{s}\right)^{r_{s}+m_{k s}}} e^{-\alpha_{s}\left\{\psi_{s}(x)\right\}} \int_{\lambda_{1}=0}^{1} \int_{\lambda_{2}=0}^{1} \lambda_{1}^{v_{1}} \lambda_{2}^{v_{2}} \lambda_{3}^{v 3} \prod_{\substack{k=1 \\
k \neq s}}^{3} \int_{\alpha_{k}=0}^{\infty} \\
& \frac{\alpha_{k}^{2 r_{k}+m_{k}}}{\left(1+\alpha_{k}\right)^{r_{k}+m_{k}}} e^{-\alpha_{k}\left\{\psi_{k}(x)\right\}} \mathrm{d} \alpha_{k} \lambda_{2} \mathrm{~d} \lambda_{1} \\
& p_{u}\left(\alpha_{s} \mid x\right)=\frac{1}{\Lambda_{u}} \sum_{j=0}^{n-r} \Phi_{u}(s) \frac{\alpha_{s}^{2 r_{s}+m_{s}}}{\left(1+\alpha_{k}\right)^{r_{s}+m_{s}}} e^{-\alpha_{s}\left\{\psi_{s}(x)\right\}}, \\
& p_{u}\left(\lambda_{1} \mid x\right)=\frac{1}{\Lambda_{u}} \sum_{j=0}^{n-r} \sum_{m=0}^{j} \sum_{m_{1}=0}^{n-r-j} \sum_{m_{2}=0}^{j-m_{1}} \sum_{m_{3}=0}^{m_{1}} \Phi \lambda_{1}^{v_{1}} \int_{\lambda_{2}=0}^{1} \lambda_{2}^{v_{2}} \lambda_{3}^{v_{3}} \prod_{k=1}^{3} \int_{\alpha_{k}=0}^{\infty} \frac{\alpha_{k}^{2 r_{k}+m_{k}}}{\left(1+\alpha_{k}\right)^{r_{k}+m_{k}}} e^{-\alpha_{k}\left\{\psi_{k}(x)\right\}} \mathrm{d} \alpha_{k} \mathrm{~d} \lambda_{2}, \\
& p_{u}\left(\lambda_{1} \mid x\right)=\frac{1}{\Lambda_{u}} \sum_{j=0}^{n-r} \sum_{m=0}^{j} \sum_{m_{1}=0}^{n-r-j} \sum_{m_{2}=0}^{j-m_{1}} \sum_{m_{3}=0}^{m_{1}} \Phi B\left(v_{2}+1, v_{1}+v_{3}+2\right) \prod_{k=1}^{3} \int_{\alpha_{k}} \frac{\alpha_{k}^{2 r_{k}+m_{k}}}{\left(1+\alpha_{k}\right)^{r_{k}+m_{k}}} e^{-\alpha_{k}\left\{\psi_{k}(x) \mathrm{d}\right.} \mathrm{d} \alpha_{k} \lambda_{1}^{v_{1}}\left(1-\lambda_{1}\right)^{v_{2}+v_{3}+1}, \\
& p_{u}\left(\lambda_{1} \mid x\right)=\frac{1}{\Lambda_{u}} \sum_{j=0}^{n-r} \Phi_{u 1} \lambda_{1}^{v_{1}}\left(1-\lambda_{1}\right)^{v_{2}+v_{3}+1}, \\
& p_{u}\left(\lambda_{2} \mid x\right)=\frac{1}{\Lambda_{u}} \sum_{j=0}^{n-r} \sum_{m=0}^{j} \sum_{m_{1}=0}^{n-r-j} \sum_{m_{2}=0}^{j-m_{1}} \sum_{m_{3}=0}^{m_{1}} \Phi \lambda_{2}^{v_{2}} \int_{\lambda_{1}=0}^{1} \lambda_{1}^{v_{1}} \lambda_{3}^{v_{3}} \prod_{k=1}^{3} \int_{\alpha_{k}=0}^{\infty} \frac{\alpha_{k}^{2 r_{k}+m_{k}}}{\left(1+\alpha_{k}\right)^{r_{k}+m_{k}}} e^{-\alpha_{k}\left\{\psi_{k}(x)\right\}} \mathrm{d} \alpha_{k} \mathrm{~d} \lambda_{1}, \\
& p_{u}\left(\lambda_{2} \mid x\right)=\frac{1}{\Lambda_{u}} \sum_{j=0}^{n-r} \sum_{m=0}^{j} \sum_{m_{1}=0}^{n-r-j} \sum_{m_{2}=0}^{j-m_{1}} \sum_{m_{3}=0}^{m_{1}} \Phi B\left(v_{1}+1, v_{2}+v_{3}+2\right) \prod_{k=1}^{3} \int_{\alpha_{k}} \frac{\alpha_{k}^{2 r_{k}+m_{k}}}{\left(1+\alpha_{k}\right)^{r_{k}+m_{k}}} e^{-\alpha_{k}\left\{\psi_{k}(x)\right\}} \mathrm{d} \alpha_{k} \lambda_{2}^{v_{2}}\left(1-\lambda_{1}\right)^{v_{1}+v_{3}+1}, \\
& p_{u}\left(\lambda_{2} \mid x\right)=\frac{1}{\Lambda_{u}} \sum_{j=0}^{n-r} \Phi_{u 2} \lambda_{2}^{v_{2}}\left(1-\lambda_{1}\right)^{v_{1}+v_{3}+1},
\end{aligned}
$$

where $s=1,2,3$,

$$
\begin{aligned}
\Phi_{u}(s) & =\sum_{m=0}^{j} \sum_{m_{1}=0}^{n-r-j} \sum_{m_{2}=0}^{j-m_{1}} \sum_{m_{3}=0}^{m_{1}} \Phi B\left(v_{1}+1, v_{2}+v_{3}+2\right) B\left(v_{2}+1, v_{1}+v_{3}+2\right) \prod_{\substack{k=1 \\
k \neq s}}^{3} \int_{\alpha_{k}} \frac{\alpha_{k}^{2 r_{k}+m_{k}}}{\left(1+\alpha_{k}\right)^{r_{k}+m_{k}}} e^{-\alpha_{k}\left\{\psi_{k}(x)\right\}} \mathrm{d} \alpha_{k}, \\
\Phi_{u 1} & =\sum_{m=0}^{j} \sum_{m_{1}=0}^{n-r-j} \sum_{m_{2}=0}^{j-m_{1}} \sum_{m_{3}=0}^{m_{1}} \Phi B\left(v_{2}+1, v_{1}+v_{3}+2\right) \prod_{k=1}^{3} \int_{\alpha_{k}} \frac{\alpha_{k}^{2 r_{k}+m_{k}}}{\left(1+\alpha_{k}\right)^{r_{k}+m_{k}}} e^{-\alpha_{k}\left\{\psi_{k}(x)\right\}} \mathrm{d} \alpha_{k}, \\
\Phi_{u 2} & =\sum_{m=0}^{j} \sum_{m_{1}=0}^{n-r-j} \sum_{m_{2}=0}^{j-m_{1}} \sum_{m_{3}=0}^{m_{1}} \Phi B\left(v_{1}+1, v_{2}+v_{3}+2\right) \prod_{k=1}^{3} \int_{\alpha_{k}} \frac{\alpha_{k}^{2 r_{k}+m_{k}}}{\left(1+\alpha_{k}\right)^{r_{k}+m_{k}}} e^{-\alpha_{k}\left\{\psi_{k}(x)\right\}} \mathrm{d} \alpha_{k} .
\end{aligned}
$$


A.2.3. Assuming Jeffreys Prior. Assuming Jeffreys prior (JP), the joint prior is

$$
\begin{aligned}
& \pi_{J}(\Omega) \propto \prod_{k=1}^{3} \frac{\left(\alpha_{k}^{2}+4 \alpha_{k}+2\right)^{1 / 2}}{\alpha_{k}\left(1+\alpha_{k}\right)} . \quad \text { (A.13) } \\
& p_{J}(\Omega \mid x)=\frac{1}{\Lambda_{J}} \sum_{j=0}^{n-r} \sum_{m=0}^{j} \sum_{m_{1}=0}^{n-r-j} \sum_{m_{2}=0}^{j-m_{1}} \sum_{m_{3}=0}^{m_{1}} \Phi \lambda_{1}^{v_{1}} \lambda v_{2}^{v_{2}} \lambda_{3}^{v_{3}} \prod_{k=1}^{3} \frac{\alpha_{k}^{2 r_{k}+m_{k}-1}}{\left(1+\alpha_{k}\right)^{r_{k}+m_{k}+1}}\left(\alpha_{k}^{2}+4 \alpha_{k}+2\right)^{1 / 2} e^{-\alpha_{k}\left\{\psi_{k}(x)\right\}},
\end{aligned}
$$

where

$$
\begin{aligned}
\Lambda_{J}= & \sum_{j=0}^{n-r} \sum_{m=0}^{j} \sum_{m_{1}=0}^{n-r-j} \sum_{m_{2}=0}^{j-m_{1}} \sum_{m_{3}=0}^{m_{1}} \Phi B\left(v_{1}+1, v_{2}+v_{3}+2\right) B\left(v_{2}+1, v_{1}+v_{3}+2\right) \prod_{k=1}^{3} \int_{\alpha_{k}} \\
& \frac{\alpha_{k}^{2 r_{k}+m_{k}-1}}{\left(1+\alpha_{k}\right)^{r_{k}+m_{k}+1}}\left(\alpha_{k}^{2}+4 \alpha_{k}+2\right)^{1 / 2} e^{-\alpha_{k}\left\{\psi_{k}(x)\right\}} \mathrm{d} \alpha_{k} .
\end{aligned}
$$

and the respective marginal posterior densities are derived as follows:

$$
\begin{aligned}
& p_{J}\left(\alpha_{s} \mid x\right)=\frac{1}{\Lambda_{J}} \sum_{j=0}^{n-r} \sum_{m=0}^{j} \sum_{m_{1}=0}^{n-r-j} \sum_{m_{2}=0}^{j-m_{1}} \sum_{m_{3}=0}^{m_{1}} \Phi \frac{\alpha_{s}^{2 r_{s}+m_{s}-1}\left(\alpha_{s}^{2}+4 \alpha_{s}+2\right)^{1 / 2}}{\left(1+\alpha_{k}\right)^{r_{s}+m_{s}+1}} e^{-\alpha_{s}\left\{\psi_{s}(x)\right\}} \int_{\lambda_{1}=0}^{1} \int_{\lambda_{2}=0}^{1} \lambda_{1}^{v_{1}} \lambda_{2}^{v_{2}} \lambda_{3}^{v_{3}} \prod_{\substack{k=1 \\
k \neq s}}^{3} \int_{\alpha_{k}=0}^{\infty} \\
& \frac{\alpha_{k}^{2 r_{k}+m_{k}-1}}{\left(1+\alpha_{k}\right)^{r_{k}+m_{k}+1}}\left(\alpha_{k}^{2}+4 \alpha_{k}+2\right)^{1 / 2} e^{-\alpha_{k}\left\{\psi_{k}(x)\right\}} \mathrm{d} \alpha_{k} \lambda_{2} \mathrm{~d} \lambda_{1} \\
& p_{J}\left(\alpha_{s} \mid x\right)=\frac{1}{\Lambda_{J}} \sum_{j=0}^{n-r} \sum_{m=0}^{j} \sum_{m_{1}=0}^{n-r-j} \sum_{m_{2}=0}^{j-m_{1}} \sum_{m_{3}=0}^{m_{1}} \Phi B\left(v_{1}+1, v_{2}+v_{3}+2\right) B\left(v_{2}+1, v_{1}+v_{3}+2\right) \prod_{\substack{k=1 \\
k \neq s}}^{3} \int_{\alpha_{k}} \\
& \frac{\alpha_{k}^{2 r_{k}+m_{k}-1}\left(\alpha_{k}^{2}+4 \alpha_{k}+2\right)^{1 / 2}}{\left(1+\alpha_{k}\right)^{r_{k}+m_{k}+1}} e^{-\alpha_{k}\left\{\psi_{k}(x)\right\}} \mathrm{d} \alpha_{k} \frac{\alpha_{k}^{\gamma_{s}-1}\left(\alpha_{s}^{2}+4 \alpha_{s}+2\right)^{1 / 2}}{\left(1+\alpha_{k}\right)^{r_{s}+m_{s}+1}} e^{-\alpha_{s}\left\{\psi_{s}(x)\right\}}, \\
& p_{J}\left(\alpha_{s} \mid x\right)=\frac{1}{\Lambda_{J}} \sum_{j=0}^{n-r} \Phi_{J}(s) \frac{\alpha_{k}^{\gamma_{s}-1}\left(\alpha_{s}^{2}+4 \alpha_{s}+2\right)^{1 / 2}}{\left(1+\alpha_{k}\right)^{r_{s}+m_{s}+1}} e^{-\alpha_{s}\left\{\psi_{s}(x)\right\}} \\
& p_{J}\left(\lambda_{1} \mid x\right)=\frac{1}{\Lambda_{J}} \sum_{j=0}^{n-r} \sum_{m=0}^{j} \sum_{m_{1}=0}^{n-r-j} \sum_{m_{2}=0}^{j-m_{1}} \sum_{m_{3}=0}^{m_{1}} \Phi \lambda_{1}^{v_{1}} \int_{\lambda_{2}=0}^{1} \lambda_{2}^{v_{2}} \lambda_{3}^{v_{3}} \prod_{k=1}^{3} \int_{\alpha_{k}=0}^{\infty} \frac{\alpha_{k}^{2 r_{k}+m_{k}-1}}{\left(1+\alpha_{k}\right)^{r_{k}+m_{k}+1}}\left(\alpha_{k}^{2}+4 \alpha_{k}+2\right)^{1 / 2} e^{-\alpha_{k}\left\{\psi_{k}(x)\right\}} \mathrm{d} \alpha_{k},
\end{aligned}
$$




$$
\begin{aligned}
& p_{J}\left(\lambda_{1} \mid x\right)=\frac{1}{\Lambda_{J}} \sum_{j=0}^{n-r} \sum_{m=0}^{j} \sum_{m_{1}=0}^{n-r-j} \sum_{m_{2}=0}^{j-m_{1}} \sum_{m_{3}=0}^{m_{1}} \Phi B\left(v_{2}+1, v_{1}+v_{3}+2\right) \prod_{k=1}^{3} \int_{\alpha_{k}} \frac{\alpha_{k}^{2 r_{k}+m_{k}-1}\left(\alpha_{k}^{2}+4 \alpha_{k}+2\right)^{1 / 2}}{\left(1+\alpha_{k}\right)^{r_{k}+m_{k}+1}} e^{-\alpha_{k}\left\{\psi_{k}(x)\right\}_{1}} \mathrm{~d} \alpha_{k} \lambda_{1}^{v_{1}}\left(1-\lambda_{1}\right)^{v_{2}+v_{3}+1}, \\
& p_{J}\left(\lambda_{1} \mid x\right)=\frac{1}{\Lambda_{J}} \sum_{j=0}^{n-r} \Phi_{J 1} \lambda_{1}^{v_{1}}\left(1-\lambda_{1}\right)^{v_{2}+v_{3}+1}, \\
& p_{J}\left(\lambda_{2} \mid x\right)=\frac{1}{\Lambda_{J}} \sum_{j=0}^{n-r} \sum_{m=0}^{j} \sum_{m_{1}=0}^{n-r-j} \sum_{m_{2}=0}^{j-m_{1}} \sum_{m_{3}=0}^{m_{1}} \Phi \lambda_{2}^{v 2} \int_{\lambda_{1}=0}^{1} \lambda_{1}^{v_{1}} \lambda_{3}^{v_{3}} \prod_{k=1}^{3} \int_{\alpha_{k}=0}^{\infty} \frac{\alpha_{k}^{2 r_{k}+m_{k}-1}\left(\alpha_{k}^{2}+4 \alpha_{k}+2\right)^{1 / 2}}{\left(1+\alpha_{k}\right)^{r_{k}+m_{k}+1}} e^{-\alpha_{k}\left\{\psi_{k}(x)\right\}} \mathrm{d} \alpha_{k} \mathrm{~d} \lambda_{1}, \\
& p_{J}\left(\lambda_{2} \mid x\right)=\frac{1}{\Lambda_{J}} \sum_{j=0}^{n-r} \sum_{m=0}^{j} \sum_{m_{1}=0}^{n-r-j} \sum_{m_{2}=0}^{j-m_{1}} \sum_{m_{3}=0}^{m_{1}} \Phi B\left(v_{1}+1, v_{2}+v_{3}+2\right) \prod_{k=1}^{3} \int_{\alpha_{k}} \frac{\alpha_{k}^{2 r_{k}+m_{k}-1}\left(\alpha_{k}^{2}+4 \alpha_{k}+2\right)^{1 / 2}}{\left(1+\alpha_{k}\right)^{r_{k}+m_{k}+1}} e^{-\alpha_{k}\left\{\psi_{k}(x)\right\}_{1}} \mathrm{~d} \alpha_{k} \lambda_{2}^{v_{2}}\left(1-\lambda_{2}\right)^{v_{1}+v_{3}+1}, \\
& p_{J}\left(\lambda_{2} \mid x\right)=\frac{1}{\Lambda_{J}} \sum_{j=0}^{n-r} \Phi_{J 2} \lambda_{2}^{v_{2}}\left(1-\lambda_{2}\right)^{v_{1}+v_{3}+1},
\end{aligned}
$$

where $s=1,2,3$,

$$
\begin{aligned}
\Phi_{J}(s)= & \sum_{m=0}^{j} \sum_{m_{1}=0}^{n-r-j} \sum_{m_{2}=0}^{j-m_{1}} \sum_{m_{3}=0}^{m_{1}} \Phi B\left(v_{1}+1, v_{2}+v_{3}+2\right) B\left(v_{2}+1, v_{1}+v_{3}+2\right) \prod_{\substack{k=1 \\
k \neq s}}^{3} \int_{\alpha_{k}} \\
& \frac{\alpha_{k}^{2 r_{k}+m_{k}-1}}{\left(1+\alpha_{k}\right)^{r_{k}+m_{k}+1}}\left(\alpha_{k}^{2}+4 \alpha_{k}+2\right)^{1 / 2} e^{-\alpha_{k}\left\{\psi_{k}(x)\right\}} \mathrm{d} \alpha_{k}, \\
\Phi_{J 1}= & \sum_{m=0}^{j} \sum_{m_{1}=0}^{n-r-j} \sum_{m_{2}=0}^{j-m_{1}} \sum_{m_{3}=0}^{m_{1}} \Phi B\left(v_{2}+1, v_{1}+v_{3}+2\right) \prod_{k=1}^{3} \int_{\alpha_{k}} \frac{\alpha_{k}^{2 r_{k}+m_{k}-1}}{\left(1+\alpha_{k}\right)^{r_{k}+m_{k}+1}}\left(\alpha_{k}^{2}+4 \alpha_{k}+2\right)^{1 / 2} e^{-\alpha_{k}\left\{\psi_{k}(x)\right\}} \mathrm{d} \alpha_{k}, \\
\Phi_{J 2}= & \sum_{m=0}^{j} \sum_{m_{1}=0}^{n-r-j} \sum_{m_{2}=0}^{j-m_{1}} \sum_{m_{3}=0}^{m_{1}} \Phi B\left(v_{1}+1, v_{2}+v_{3}+2\right) \prod_{k=1}^{3} \int_{\alpha_{k}} \frac{\alpha_{k}^{2 r_{k}+m_{k}-1}}{\left(1+\alpha_{k}\right)^{r_{k}+m_{k}+1}}\left(\alpha_{k}^{2}+4 \alpha_{k}+2\right)^{1 / 2} e^{-\alpha_{k}\left\{\psi_{k}(x)\right\}} \mathrm{d} \alpha_{k} .
\end{aligned}
$$

\section{Data Availability}

Previously reported data were used to support this study and are available at https://www.nilebasin-journal.com/images/ files/uploads/3047_20093751.pdf, DOI: 10.9734/BJMCS/ 2014/9193. The prior studies (and datasets) are cited at relevant places within the text as references [23] and [24].

\section{Conflicts of Interest}

The authors have no potential conflicts of interest, such as financial interests, affiliations, or personal interests or beliefs that could be perceived to affect the objectivity or neutrality of the article.

\section{References}

[1] P. Kumaraswamy, "A generalized probability density function for double-bounded random processes," Journal of Hydrology, vol. 46, no. 1-2, pp. 79-88, 1980.

[2] J. Mazucheli, A. F. B. Menezes, and S. Chakraborty, "On the one parameter unit-lindley distribution and its associated regression model for proportion data," Journal of Applied Statistics, vol. 46, no. 4, pp. 700-714, 2019.

[3] A. Biswas and S. Chakraborty, "R=P $(Y<X)$ for unit-lindley distribution: inference with an application in public health," 2019, https://arxiv.org/abs/1904.06181.

[4] S. Nadarajah and S. Chan, "On moments of the unit Lindley distribution," Journal of Applied Statistics, vol. 47, no. 5, pp. 947-949, 2020.

[5] E. Alton and G. M. Cordeiro, "The unit-improved seconddegree Lindley distribution: inference and regression modeling," Computational Statistics, vol. 35, no. 1, pp. 259-279, 2020.

[6] H. T. K. Akdur, "Unit-lindley mixed-effect model for proportion data," Journal of Applied Statistics, vol. 2020, pp. 1-17, 2020.

[7] A. Frigessi, O. Haug, and H. Rue, "A dynamic mixture model for unsupervised tail estimation without threshold selection," Extremes, vol. 5, no. 3, pp. 219-235, 2002.

[8] F. F. do Nascimento, D. Gamerman, and H. F. Lopes, "A semiparametric bayesian approach to extreme value estimation," Statistics and Computing, vol. 22, no. 2, pp. 661-675, 2012. 
[9] R. S. Jagtap and U. V. Naik-Nimbalkar, "Modelling unusual behaviour of rainfall using truncated GEV distribution in a mixture framework," Asian Journal of Water Environment and Pollution, vol. 18, no. 1, pp. 31-41, 2021.

[10] K. S. Sultan, M. A. Ismail, and A. S. Moisheer, "Mixture of two inverse Weibull distributions: properties and estimation," Computational Statistics \& Data Analysis, vol. 51, no. 11, pp. 5377-5387, 2007.

[11] A. S. Hassan, A. M. Elfattah, and M. M. Hassan, "Bayesian analysis for mixture of burr XII and burr X distributions," Far East Journal of Mathematical Sciences, vol. 103, no. 6, pp. 1031-1041, 2018.

[12] N. Feroze and M. Aslam, "Three-component mixture of Rayleigh model under doubly censored samples: a bayesian look," Communications in Mathematics and Statistics, vol. 7, no. 4, pp. 417-443, 2019.

[13] A. S. Al-Moisheer, A. F. Daghestani, and K. S. Sultan, "Mixture of Lindley and lognormal distributions: properties, estimation, and application," Journal of Function Spaces, vol. 2021, no. 1, pp. 1-12, 2021.

[14] M. Aslam, R. Yousaf, and S. Ali, "Two-component mixture of transmuted fréchet distribution: bayesian estimation and application in reliability," Proceedings of the National Academy of Sciences, India - Section A: Physical Sciences, vol. 91, no. 2, pp. 309-336, 2021.

[15] A. N. Cheema, M. Aslam, I. M. Almanjahie, and I. Ahmad, "Bayesian modeling of 3-component mixture of exponentiated inverted Weibull distribution under noninformative prior," Mathematical Problems in Engineering, vol. 2020, pp. 1-11, 2020.

[16] M. Khalid, M. Aslam, and T. N. Sindhu, "Bayesian analysis of 3-components Kumaraswamy mixture model: quadrature method vs. Importance sampling," Alexandria Engineering Journal, vol. 59, no. 4, pp. 2753-2763, 2020.

[17] J. B. Kadane, "Predictive and structural methods for eliciting prior distributions," in Bayesian Analysis in Econometrics and Statistics: Essays in honor of Harold Jeffreys, A. Zellner, Ed., pp. 89-93, North Holland Publishing Company, Amsterdam, Netherlands, 1980.

[18] P. H. Garthwaite and J. M. Dickey, "Elicitation of prior distributions for variable-selection problems in," Regression. The Annals of Statistics, vol. 20, no. 4, pp. 1697-1719, 1992.

[19] M. Aslam, "An application of prior predictive distribution to elicit the prior density," Journal of Statistical Theory and Applications, vol. 2, no. 1, pp. 70-83, 2003.

[20] E. D. Hahn, "Re-examining informative prior elicitation through the lens of Markov chain Monte Carlo methods," Journal of the Royal Statistical Society: Series A, vol. 169, no. 1, pp. 37-48, 2006.

[21] J. Mazucheli, A. F. B. Menezes, and S. Chakraborty, "Discussion comments on 'on moments of the unit lindley distribution'," Journal of Applied Statistics, vol. 47, no. 5, pp. 950-953, 2020.

[22] J. G. Norstrom, "The use of precautionary loss functions in risk analysis," IEEE Transactions on Reliability, vol. 45, no. 3, pp. 400-403, 1996.

[23] M. M. Abdel-Latif and M. Yacoub, "Effect of change of discharges at dongola station due to sedimentation on the water losses from nasser lake," Nile Basin Water Science \& Engineering Journal, vol. 4, no. 1, pp. 86-98, 2011.

[24] M. El-Deen, G. R. Al-Dayian, and A. A. El-Helbawy, "Statistical inference for Kumaraswamy distribution based on generalized order statistics with applications," British Journal of Mathematics \& Computer Science, vol. 4, no. 12, pp. 1710-1743, 2014. 